\title{
Speakable and Unspeakable, Past and Future
}

\author{
Aephraim M. Steinberg \\ Department of Physics \\ University of Toronto \\ Toronto, ONT M5S 1A7 \\ CANADA \\ (version of October 30, 2018)
}

\subsection{Introduction}

A volume in honour of a visionary thinker such as John Archibald Wheeler is a rare license to exercise in the kind of speculation and exploration for which Wheeler is famous, but which most of the rest of us usually feel we had better keep to ourselves. We have all - even those of us who never had the fortune to work directly with him - been inspired and motivated by Wheeler's creativity and open-mindedness. For all of our apparent understanding of quantum mechanics, our ability to calculate remarkable things using this theory, and the regularity with which experiment has borne out these predictions, at the turn of the 21st century it seems there are as many puzzles on the road to a true understanding of quantum theory as there were at the start of the previous century. Then, at least, one could hope to be guided by the mysteries of unexplained experiment. Now, by contrast, we may seem to have lost our way, as even though our experiments are all "explained" (in some narrow sense which can only be deemed satisfactory out of fear to leap beyond the comfortable realm of formalism), the theory itself is mysterious. Further explorations, without the anchor of experiment, certainly run the risk of becoming mere flights of metaphysical fancy, giving rise to factions characterized less by intellectual rigor than by fundamentalist zeal. Yet it would be premature to give up the journey before at least trying to establish foothold on the terrain ahead. Following Wheeler's example, we can invent new experiments to help us speak about some of the unspeakable aspects of our theory, and to venture forward.

I have therefore decided to use this occasion to describe a number of 
loosely connected ideas we have been thinking about and experiments we have been working on in my group, which I believe relate to deep questions about how one should understand quantum mechanics. In keeping with the best tradition, I provide no answers to these questions, but I hope that I can show how a variety of questions are related to one another, and related to experiments both gedanken and real. Everything which follows takes place in the setting of standard quantum theory, and therefore even the most surprising predictions or observations I discuss are of course unambiguous, and implicit in every quantum textbook. Why then are they surprising? Clearly, we are not surprised only by results which contradict our theories; as is obvious when one discusses classical physics with students learning it for the first time, we are surprised by results which contradict what we understand of these theories. Over and over again in the past decade or two, experiments in fields such as quantum optics have revealed phenomena which surprise even those of us who ought by now to know quantum theory reasonably well. While many thinkers seem to consider such experiments mere parlor tricks, does not the ability of these experiments to evoke continued surprise demonstrate that we still do not understand quantum theory the way we understand classical theory? This simple observation is so clichéd as to bear repeating, for too many physicists have fallen prey to the reassuring but nihilistic thesis that since so many before us have failed, we would be wasting our time to seek any deeper understanding of quantum theory than is contained in our beautiful equations.

\subsection{Past and future, particle and wave, locality and nonlocality}

"Prediction is difficult, especially of the future."

This famous phrase is generally attributed to Yogi Berra, although among scientists one hears the credit given to Niels Bohr with some frequency. While the latter attribution has a certain comforting believability to it, one wonders whether Bohr's theory would make the past any more amenable to analysis than the future. A moment's thought suffices to realize that as difficult as prediction of the future may be, prediction of the past is not necessarily any easier (even aside from the semantic issue, which leads us to adopt the term "retrodiction" for inferences about the past). Neither is more or less the domain of science, although physics has traditionally concentrated on prediction while 
fields such as archaeology and cosmology have dealt with retrodiction. In classical mechanics, nevertheless, time-reversal symmetry guarantees that retrodiction is precisely the same task as prediction $\dagger$. But in quantum mechanics as it is generally taught, despite the time-reversibility of the Schrödinger equation, retrodiction appears particularly mysterious. If I fire a photon towards a double-slit, quantum mechanics unambiguously tells me what the state of the photon is after passing through the slits, although this state only gives probabilities for individual measurement outcomes. But when I see the photon land at a particular point on the screen (see Fig. 1), what can I conclude about which slit it went through? The usual approach to measurement, involving an uncontrollable, irreversible disturbance, effectively decouples the "collapsed" state from what came before, except insofar as the probabilities for the measurement results are determined by the initial state. This is quite different from the usual treatment of state preparation, which sets up a well-defined initial condition and allows unitary evolution to take over.

The orthodox view of quantum mechanics holds that what has been measured can be known, and what has not is "unspeakable." If a particle is prepared in a certain wave packet, that function is to be considered a complete description, and any additional questions about where the particle "ist" are deemed uncouth, at least until such a measurement is made. The absence of trajectories in quantum mechanics means that one supposedly has no right to discuss where the particle "was" prior to that measurement. Yet the fundamental laws of quantum mechanics are as time-reversible as those of Newton, and one quite reasonably wonders why it is any less valid to use a measurement to draw inferences about a particle's history than to make predictions as to its future behaviour. Such considerations led Yakir Aharonov and his coworkers to a formalism of "weak measurements" which allows one to discuss the state of evolving quantum systems in a fundamentally time-symmetric way. This chapter draws heavily on their ideas, whose main elements I will introduce below. I will analyze how weak measurements can be applied to several experimentally interesting situations. Consider, for one example, the problem of a tunneling particle. What can we know about where a particle was before it appeared on the far side of a forbidden barrier? Is it ever localized in the "forbidden" region? Can we obtain

$\dagger$ For closed systems, at any rate- the thermodynamic arrow of time breaks the symmetry in the case of open systems.

$\dagger$ Indeed, I once received an anonymous referee report which read, in essence, "This work is interesting, but I am unsure what the author means by the word 'is'." 
more information about the particle's history from the state preparation, or from the observation that it was transmitted?

These new ideas about measurement naturally lead one to think about epistemology. Is the wave function the fullest description of what we can know about a system? Is there then a real sense in which a particle may be in two places at the same time? Can we sometimes have more information than is encoded in a single wave function, by utilizing pre- and post-selection simultaneously? Or, on the contrary, is it impossible even to know as much as a wave function, and are we limited to knowing the outcomes of the specific measurements we have performed? Can we have anything more than statistical knowledge about the outcomes of future measurements? Some experiments we plan to perform are designed to touch on these issues. In addition, they make one question whether even our probabilistic description of reality is complete, or whether exotic entities such as negative or complex probabilites may actually be meaningful.

The explosive growth of the field of quantum information, with its potential applications and headline-making buzzwords, has surprised many by turning "philosophical" research programmes into timely, relevant, and some suspect even lucrative projects. These questions about past and future are no exception. Some of our recent work has involved the development of a quantum "switch," in which a single photon may be transmitted or not, depending on whether or not a single other photon is present. The thorn is that it is impossible to know whether either photon was ever present in the first place... as in many quantum optics experiments, the outcome depends on conditions which can only be measured after the fact. On this new work, I have no philosophical conclusions to draw: only a cautionary tale about how tricky these quantum conundra remain even for those building the experiments, and a hope that others will help us learn how to think about our own experiments in new ways.

To come full circle, our first planned application of this "switch" is to carry out an experimental investigation of quantum reality first proposed by Lucien Hardy, extending ideas due to Elitzur and Vaidman. This experiment allows one to demonstrate that what at first glance appears to be perfectly airtight reasoning about the history of particles once they have been detected can lead to a seeming contradiction. More recently, it has been recognized that this contradiction can be eliminated if one applies the formalism of weak measurements and accepts these "exotic" 
probabilities as a correct description of reality. We believe that most if not all of these ideas are now accessible in the laboratory.

\subsection{Weak measurements}

The question of what measurement is is of course one of those which has haunted quantum theory from the start. Why does one thing occur and not another (let alone more than one)? When is a measurement? How does this relate to the arrow of time? By thinking carefully about retrodiction as well as prediction, some of these issues can be, if not resolved, then perhaps at least brought into starker relief. Aharonov et al. have led the way in generalising concepts of measurement in this direction (Aharonov and Vaidman, 1990), with their formalism of "weak measurement." In particular, weak measurements allow one to put past and future on an equal footing- and, better yet, to do something which is commonplace to any experimentalist and yet seemingly at odds with the usual machinery of quantum theory: to use one's knowledge of the initial and the final conditions of a system together to draw conclusions about what came in between.

If the task of deducing what happened before a measurement was made based on the result of that single measurement seems to conflict with the standard prescriptions of quantum theory, this is because the measurement is postulated to irrevocably change the state of the system. But what is the origin of this disturbance? Let us leave aside any considerations of "collapse" for the time being, and think only about the effect of an interaction between some system to be studied, and some other quantum mechanical system which will serve as a "pointer," or measuring device. Amplification of the state of this pointer to the macroscopic realm, so that a human observer might take note of it, can happen at some later stage if necessary; for our purposes, the important questions about measurement can all be treated simply by considering the effects of this quantum mechanical interaction.

In the standard approach due to von Neumann von Neumann, 1955, 1983), a measurement of a system observable $A_{s}$ can be effected via an interaction Hamiltonian

$$
\mathcal{H}=g(t) A_{s} \cdot P_{p}
$$

where the time-dependence $g(t)$ allows the measurement to take place during a finite interval of time, and where $P_{p}$ is the canonical momentum 
of the pointer. Since the momentum is the generator of spatial translations, the effect of this interaction is to displace the pointer position by an amount proportional to the value of $A_{s}$. In particular, for suitably normalized $g(t)$, the expectation value of the pointer position will change by an amount which is proportional to the expectation value of $A_{s}$, and thus serves as a record of this measured value. Naturally, the requirement for a "good" measurement is that the pointer position be sufficiently well-defined that for different eigenvalues of $A_{s}$, the final state of the pointer is measurably different $\dagger$. In this case, the pointer and the system become entangled, and the irreversibility of the measurement can be seen as arising from the effective decoherence of the system wave function when one traces over the state of the pointer.

The back-action on the system can be seen in another way, which is that the above Hamiltonian exerts an uncertain force on the system, to the extent that $P_{p}$ is uncertain. If the pointer were in an eigenstate of momentum, then the measurement interaction would be an entirely predictable, unitary evolution of the system, $\mathcal{H} \propto A_{s}$; no irreversibility would thereby be introduced. Of course, if the pointer momentum were perfectly well defined, the pointer position would be entirely uncertain, and it would be impossible to observe a translation of the pointer. No measurement would have occurred.

Aharonov et al. argue that it is reasonable to consider an intermediate regime, where some information is captured during a measurement interaction, yet where the disturbance on the system is limited. Although this is not the textbook model of a quantum measurement, it is in fact a good model of how countless experiments are actually performed. Frequently, measurements on individual systems have such large uncertainties that only by averaging over thousands or millions of trials can statistical information be extracted.

The theoretical idea of a "weak measurement" is then to carry out a von Neumann interaction, but with an initial pointer state which is so delocalized in position that no single measurement can determine with certainty the value of $A_{s}$. On the flip side, this pointer may have such small uncertainty in momentum that the back-action on the system can be made arbitrarily small. It is in fact straightforward to verify that under these conditions, instead of entangling the system and pointer

$\dagger$ Clearly, in the case of an observable with a continuous spectrum at least, one must be more cautious in defining precisely which eigenvalues ought to be distinguishable. 
according to

$$
|\Psi\rangle_{s} \phi_{p}(x) \rightarrow \sum_{i} c_{i}\left|\psi_{i}\right\rangle_{s} \phi_{p}\left(x-g a_{i}\right)
$$

(where the $\psi_{i}$ and $c_{i}$ are the eigenkets of $A_{s}$ and their corresponding amplitudes, and $g a_{i}$ is the shift in the pointer wavefunction $\phi_{p}$ which corresponds to an eigenvalue $a_{i}$ ), the system and pointer remain to lowest order unentangled:

$$
|\Psi\rangle_{s} \phi_{p}(x) \rightarrow|\Psi\rangle_{s} \phi_{p}\left(x-g\left\langle A_{s}\right\rangle\right) .
$$

On average, the pointer is displaced by an amount related to the expectation value of $A_{s}$, but since this shift is too small to significantly modify the pointer state, the system is unaffected.

Importantly, this means that the original evolution of the particle may continue, and one may ask not only about the correlations between the pointer position and the initial state of the system, but equally well about correlations between the pointer position and the state the system is later observed to be in. One may quite generally ask what will happen to the pointer on those occasions where the system was prepared in state $|i\rangle$ before the measurement interaction, and later measured to be in some final state $|f\rangle$. Using standard quantum theory, Aharonov and coworkers showed that the mean shift of the pointer position for this subensemble corresponds to a "weak value" of $A_{s}$ given by

$$
\left\langle A_{s}\right\rangle_{\mathrm{wk}}=\frac{\left\langle f\left|A_{s}\right| i\right\rangle}{\langle f \mid i\rangle} .
$$

Clearly, for the trivial case $f=i$, this reduces to the usual expression for an expectation value. But for the more general case, it is heartening to note that the initial and final states have equal importance for the measured value of $A_{s}$; one can learn as much about a particle's state by observing its future as by knowing its past.

There are many other striking properties of weak measurements which suggest that they are a powerful tool for analyzing a broad variety of physical situations, and also that there may be some deep physical meaning to these quantities themselves. I will not go over these in detail, but (Reznik and Aharonov, 1995) and (Aharonov and Vaidman, 2002a) provide a deep analysis. In many ways, these values can be seen as a natural application of Bayesian probability theory to quantum mechanics (Steinberg, 1995a b) , satisfying many of the natural axioms of probability theory. More important, they describe the outcomes of any 
measurements which can be described using the (modified) von Neumann formalism, and therefore show a clear connection to physical observables, not to mention a unifying framework within which a broad class of experiments may be treated. At the same time, they display a number of troubling features. Notably, the measured weak value need not be consistent with any physically plausible values of $A_{s}$; it need not even fall within this operator's eigenvalue spectrum. More shocking still, some positive-definite quantities such as energy (or even probability) may be measured to be negative (Aharonov et al., 1993a). In fact, weak values are in general complex numbers rather than reals. As explained in some of the above references, this is not an entirely untenable state of affairs, and the physical significance of the real and imaginary parts of the weak value may be clearly identified. Roughly speaking, the real part indicates the size of the physical shift in pointer position, the measurement result one expected classically from such a device. The imaginary part indicates how much the momentum of the pointer will change as an unintended consequence of the measurement interaction, and consequently, how large the back-action of the measurement on the system.

One of the truly exciting features of weak measurements is that simultaneous weak measurements may be made on non-commuting observables, and do not render each other impossible, or even modify each other's results. For instance, if a particle is prepared in a eigenstate of some operator $B$ with eigenvalue $b_{j}$, then a weak measurement of $B$ is guaranteed to yield the value $b_{j}$, regardless of the postselection. Similarly, if it is postselected to have an eigenvalue $c_{j}$ of some operator $C$, then a weak measurement of $C$ is certain to yield $c_{j}$, regardless of the preparation. If both $B$ and $C$ are measured weakly between the preparation and the postselection, both of these values will be observed (albeit as average shifts of a very uncertain pointer position)- even if $B$ and $C$ do not commute. For that matter, if $B+C$ is measured, the result will be $b_{j}+c_{j}$, something which makes intuitive "classical" sense, but which one could never hope for in the context of strong quantum measurements. Such properties clearly hold out the tantalizing possibility of making more of reality "speakable" (in John Bell's term (Bell, 1987)) than we are usually led to believe. When we think about a particular system which survived from state preparation through postselection, should we merely think of the initial state evolving in a unitary fashion until the postselection induced a collapse, or should we think about its properties as depending on both pre- and post-measurements? While the orthodox 
view may be that if no measurement is performed between preparation and postselection, the question is meaningless, it is thought-provoking that any von Neumann-style interaction which takes place at intermediate times, provided that it is not so strong as to irreversibly modify the system dynamics, will produce an effect whose magnitude is defined by this new formalism. Such observations led to a variety of speculations about the "reality of the wave function" Aharonov and Anandan, 1993b) and to a general formulation of quantum mechanics via "two-time wave functions" (Reznik and Aharonov, 1995).

Recently, a connection has been drawn between weak measurements and more widespread techniques for dealing with the quantum evolution of open systems (Wiseman, 2002), and this has proved useful for explaining the "negative-time correlations" in a cavity QED experiment (Foster et al., 2000). Specifically, an experiment in Luis Orozco's group designed to observe the evolution of an electromagnetic field after the detection of one photon also found interesting dynamics in the evolution of the field before the detection of a photon. Howard Wiseman pointed out that when the photodetection event is treated as a postselection, an extension of weak measurement theory can be fruitfully applied to understand this negative-time evolution, which had not previously been fully explained.

\subsection{A quantum-mechanical shell game}

While it was recognized from the outset that weak measurements could yield anomalously large values, and the first (intentional!) experimental implementation of weak measurements was a linear-optics experiment to demonstrate how a spin measurement could yield an apparently nonsensical value (Ritchie et al., 1991), it was pointed out (Steinberg, 1995b) that there is a striking mathematical relationship between weak measurements and classical probability theory. In fact, the result of Eq. 0.4 can be obtained quite generally by summing over the "conditional probabilities" for each of the eigenstates of the operator,

$$
\langle A\rangle_{\mathrm{wk}}=\sum_{j} a_{j} P(j \mid i, f),
$$

where the probability of being in an eigenstate $\left|\psi_{j}\right\rangle$ is defined as the expectation value of the projector $\left|\psi_{j}\right\rangle\left\langle\psi_{j}\right|$; the "conditional probability" is the natural generalisation based on the weak-measurement prediction

for the shift experienced by a pointer which couples to this projection 
operator, conditioned on the appropriate post-selection:

$$
P_{\mathrm{wk}}(j \mid i, f)=\frac{\left\langle f \mid \psi_{j}\right\rangle\left\langle\psi_{j} \mid i\right\rangle}{\langle f \mid i\rangle} .
$$

Of course, these conditional probabilities sometimes prove to have values greater than 1 , less than 0 , or even with imaginary components. It is on the one hand unclear if it is meaningful in any real sense to interpret these as probabilities, while on the other hand the weak-value expressions for probability are defined in clear analogy to classical probabilities, and satisfy the same axioms. Furthermore, the experiments which are predicted to yield negative or complex "probabilities" are designed in precisely the fashion one would choose classically to measure the conditional probabilities, and they would correctly measure these probabilities when used in the classical regime; is this not the operational prescription for developing the quantum mechanical formalism for a given observable?

I do not possess the hubris to attempt to pronounce a final verdict on how seriously one should take these probabilities, or on whether one would be better to avoid such a loaded term at all. Nevertheless, the expressions derived in this fashion have clear physical significance for a wide-ranging class of experiments. Suffice it to note that there are a number of other contexts (such as "rescuing" locality, in the context of Bell's theorem) in which other authors have suggested taking seriously the concept of negative probability in quantum mechanics Muckenheim et al., 1983; Fevnman, 1987; Pitowski, 1982; Scullv, 1994), not to mention the negative quasiprobabilities which are familiar in the context of the Wigner function and other phase-space distributions (Wigner, 1932; Liebfried et al., 1996).

Let us for now accept this terminology of probabilities, with all its caveats, and examine some striking examples of what weak-measurement theory predicts. In 1991, Aharonov and Vaidman applied the formalism to the following toy problem (Aharonov and Vaidman, 1991). Consider a particle which can be in any of three boxes, which we will denote as three orthogonal states $|A\rangle,|B\rangle$, and $|C\rangle$. Let us prepare the particle in an initial state

$$
|i\rangle=\frac{|A\rangle+|B\rangle+|C\rangle}{\sqrt{3}},
$$

i.e., a symmetric equal superposition of being in each of the three boxes. Suppose that some time later we choose another basis, and measure 
whether or not the particle is in the final state

$$
|f\rangle=\frac{|A\rangle+|B\rangle-|C\rangle}{\sqrt{3}},
$$

where the sign in front of box $C$ has been changed. Note that there is some probability for this postselection to succeed, without any need for the particle to change its state between the measurements; $|\langle f \mid i\rangle|^{2}=$ $1 / 9$.

Obviously, the question of interest is how we should describe the state of the particle between the state-preparation and a successful postselection. Should we evolve $|i\rangle$ forward in time under the free Hamiltonian, the particle remaining symmetrically distributed among the three boxes, until the final measurement disturbs its phase? Or should we instead evolve $|f\rangle$ backwards in time? Clearly, orthodox quantum mechanics says there is no meaning to the question of at what time $C$ stopped being in phase with $A$ or $B$, and began being out of phase with them; Bohr would tell us that the value of this phase during a period when nothing in the apparatus is sensitive to it is meaningless. Similarly, we cannot ask which of the three boxes the particle was in before it was detected in $|f\rangle$, although it seems quite natural to suppose it had equal probabilities to be found in any of them.

One can conceive of measuring such probabilities, by using a large ensemble of particles. For instance, a test charge held near box A may experience a slight momentum shift if and only if the particle is in box A. If this shift is arranged to be far smaller than the uncertainty in the test charge's momentum, then it may be possible to carry out such measurements without any appreciable effect on the evolution of the particle. If no postselection is performed, the magnitude of this shift will be proportional to the probability that the particle was indeed in $A$, i.e., the expectation value of the projection operator $|A\rangle\langle A|$. For the state $|i\rangle$, for instance, this probability is one third: the impulse imparted to the test charge after $N$ particles go through the boxes will be precisely what one would expect if $N / 3$ had been in box A...or, equivalently, if one third of each of the $N$ particles had been in box $A$.

What if the momentum shift on the test charge is recorded (including its large uncertainty) each time a particle passes, but is discarded unless the postselection fails? Then the sum of the momentum shifts for all the test charges which interacted with particles eventually detected in $|f\rangle$ will describe the conditional probability that those particles had been in 
box $A$ :

$$
\begin{aligned}
P_{\mathrm{wk}}(A \mid i, f) & =\frac{\langle f|\operatorname{Proj}(\mathrm{A})| i\rangle}{\langle f \mid i\rangle} \\
& =\frac{\langle f \mid A\rangle\langle A \mid i\rangle}{\langle f \mid i\rangle} .
\end{aligned}
$$

It is easy to verify that this probability is unity. The postselected test charges will display precisely the same mean momentum shift as they would for a particle prepared with $100 \%$ certainty in box $A$. Similarly, the weak (or conditional) probability for the particle to be in box $B$ is $100 \%$. And the axioms of probability? Must not the probabilities of all the exclusive possibilities add up to 1 ? Indeed- it is equally easy to verify that $P_{\mathrm{wk}}(C \mid i, f)$, the conditional probability for a particle to have been in box $C$ between its preparation in $|i\rangle$ and its detection in $|f\rangle$, is -1 . Meaningless? Not at all. If the mean momentum shift of test charges which interact with particles eventually detected in state $|f\rangle$ is measured, it will be found to have the "wrong" sign- that is, if the particle and the test particle have charges of like signs and ought to repel each other, the test charge will be found to have a mean momentum towards box $C$. Perhaps it is risky to interpret this by saying the particle truly had a negative probability to be in that box- yet physically, its effect was equal and opposite to the effect of a particle in box $C$.

Perhaps more striking yet is the observation that the particle was "definitely" in box $A$, but also in box $B$. We are quite accustomed to saying that a particle must go through "both slits at once" in Young's interferometer, but how many of us truly mean it? The wave function, of course, traverses both slits, but we know full well that to talk of "the" position of the particle, we must introduce some position-measurement, in which case the particle will be observed at one slit or the other. Weak measurements show us that this is not necessarily always the case, so long as no "collapse" (or decoherence, more precisely) is introduced during the measurement. Aharonov et al. have used these features of the theory to argue in favour of the ontological "reality of the wave function" (Aharonov and Anandan, 1993b), while these arguments have incited a great deal of controversy (Unruh, 1994). More recently, Aharonov and Vaidman have tried to respond to some objections to their shell-game paper by introducing a strong measurement- they show that if this particle is a "shutter," then a photon heading towards either box $A$ or box $B$, or indeed any superposition of the two, is guaranteed to be intercepted by the shutter (in cases where the shutter is postselected to be in 
$|f\rangle$, as always (Aharonov and Vaidman, 2002b). This suggests that the nonlocality of quantum mechanics may be even deeper than usually recognized, in that a given particle could actually have measurable effects in two places at the same time.

We are currently setting up an experiment, shown schematically in Figure 2, designed to test some of the features of this quantum conundrum. Photons are prepared in a symmetric superposition of the three "boxes" $A, B$, and $C$, by the use of beam-splitters; each box is in fact one path in an interferometer. By carefully adjusting the relative phases of the paths (specifically, by introducing an extra $\pi$ phase shift along path $C$ before symmetrically recombining the three beams at another beam splitter), it is possible to project out light in the state $[|A\rangle+|B\rangle-|C\rangle] / \sqrt{3}$. Several varieties of weak measurement may be performed. In particular, a small piece of glass can introduce a spatial shift in one of the three beams, smaller than the width of the beam (i.e., the uncertainty in the photon's transverse position). Alternatively, a waveplate can rotate the polarisation of one of the paths by a small angle. It is an optics problem left for the reader to show that the deviations to be expected are precisely those predicted by weak measurement theory: if beam $A$ or $B$ is displaced by $\delta x$, then the output will be displaced by $\delta x \ldots$ on the other hand, if beam $C$ is displaced by the same amount, the displacement at the output will be $-\delta x$. (In the optics context, it is not difficult to understand this as an interference effect related to the $\pi$ phase shift introduced in $\operatorname{arm} C$.) We plan not only to confirm the weakmeasurement predictions, but also to study the correlations between the different probabilities. In particular, we are interested in the question of nonlocality. If we can say with certainty that the particle was in $A$ and that it was in $B$, can we also say that it was simultaneously in $A$ and $B$ ? This may seem obvious, but again, with weak measurements one must be careful.

In their paper on "How One Shutter Can Close $N$ Slits" Aharonov and Vaidman, $2002 \mathrm{~b}$ ), Aharonov and Vaidman note that a pair of test particles, one heading to shutter position $A$ and the other to shutter position $B$, could not both be reflected by a single shutter (although they make interesting observations about the case of multiple slits, multiple shutters, and multiple incident particles). In essence, the reflection of a particle heading towards $A$ is a strong measurement, and prevents the slit from stopping a second particle heading towards $B$. However, one can put this even 
more succinctly if one accepts the definition

$$
P(A \& B)=\langle\operatorname{Proj}(\mathrm{A}) \cdot \operatorname{Proj}(\mathrm{B})\rangle .
$$

Although this definition has certain pathologies associated with it Steinberg, 1995b) (notably, this product of two projectors need not be a Hermitian operator, and therefore could yield complex "joint probabilities" even in non-post-selected systems), it seems the most natural way of describing joint probabilities. It generalizes easily to the case of weak (conditional) measurements. However, if $A$ and $B$ are orthogonal, as in the present case, then the product of their projectors

$$
\begin{aligned}
& \operatorname{Proj}(\mathrm{A}) \cdot \operatorname{Proj}(\mathrm{B})=|A\rangle \quad\langle A \mid B\rangle \quad\langle B| \\
& =|A\rangle \quad 0 \quad\langle B|=0 \text {. }
\end{aligned}
$$

Under no circumstances is there a nonzero joint probability, conditional or otherwise, to be in box $A$ and to be in box $B$. As discussed in Aharonov et. al, 2002), weak measurements do not allow one to conclude that because $P(A)=P(B)=1$, then $P(A \& B)$ must also be 1 ; this is because the probabilities themselves are not bounded by 0 and 1 . The probability of "A and B" may vanish, in spite of the certainty of $A$ and $B$ individually, for the probability of "A and not $\mathrm{B}$ " is one. If this seems strange, given that the probability of "not B" is zero, no worries: for the probability of "not A and not B" is negative 1. This odd state of affairs is summarized in table 1.

\subsection{Tunneling}

Another problem where nonlocality has been a topic of discussion in recent years is that of tunneling through a barrier. It has been well-known since early in the century (Wigner, 1995; MacColl, 1932; Hauge and Støvneng, 1989: Büttiker and Landauer, 1982) that the group delay (stationary phase time) for a wave packet incident on an opaque barrier of thickness $d$ to appear on the far side saturates to a finite value as $d$ tends to infinity. For large enough $d$, this implies superluminal propagation speeds for the peak of the wave packet, which naturally provoked much skepticism. A number of experiments, including one I performed along with Paul Kwiat in Ray Chiao's group at Berkeley (Steinberg et al., 1993), demonstrated that this prediction is indeed correct (Enders and Nimtz, 1993; Spielmann et al.,1994), although no violation of causality is implied (Chiao and Steinberg, 1997). Due to the difficulty of timing the arrival of matter particles 


\begin{tabular}{cccc} 
Probabilities & A & not A & A or not A \\
\hline \hline B & 0 & 1 & 1 \\
\hline not B & 1 & -1 & 0 \\
\hline \hline B or not B & 1 & 0 &
\end{tabular}

Fig. 0.1. This table summarizes the probabilities and joint probabilities of finding the particle in or out of box $A$ and in or out of box $B$, demonstrating how a negative probability in one column can allow the joint probability of two "certain" events to vanish.

through any reasonable tunnel barrier, and the problems of reaching the relativistic regime with massive particles, these experiments were carried out with photons. We are now building at Toronto a series of experiments designed to observe the tunneling of laser-cooled atoms through micron-scale barriers formed by focussed beams of light Steinberg et al., 1998a: Steinberg, 1998b). Although the experiments are complex, this should open up a broad new vista of phenomena to study. In particular, it becomes possible to probe the particles while they are traversing the "forbidden" region, and also to study the effects of decoherence on the tunneling process (Steinberg, 1999a).

While it is certainly strange that a wave packet peak should arrive in less time than if the original peak had travelled at the speed of light, it was pointed out comparitively early in the (latest bout of the) tunneling time controversy that no physical law guarantees any direct causal connection (let alone identity) between an incoming peak and an outgoing peak (Büttiker and Landauer, 1982). We generally interpret these effects as remarkable but entirely causal "pulse reshaping" phenomena, in which the leading edge of a pulse is preferentially transmitted, while its trailing edge is preferentially reflected, thus biasing the peak towards earlier times. Similar effects had been observed in the 1980s in the context of propagation through absorbing media (Garrett and McCumber, 1970; Chu and Wong, 1982), and much excitement has recently been created by the analogous observation of faster-than-light propagation in transparent (but active) media (Steinberg and Chiao, 1994; Wang et al., 2000: Steinberg, 2000). A review of superluminality and causality in optics is given in (Chiao and Steinberg, 1997).

These counter-intuitive effects occur only when the tunneling probability is relatively small. In other words, like many weak-measurement paradoxes, the anomalies are dependent on the success of a postselec- 
tion which occurs only rarely. If one tracks the centre of mass of a wave packet incident on a tunneling barrier, it never moves faster than lightonly when one projects out the transmitted portion alone does the peak abruptly appear to have travelled superluminally. In this sense, one may well argue that the superluminality is not a function of propagation through the tunnel barrier, but only of this mysterious "collapse" event whereby a particle previously spread out across two peaks may choose to localize itself on one. Nevertheless, it seems reasonable to apply the formalism of weak measurements to the tunneling problem, in order to see whether this can shed light on the counterintuitive aspects of the situation. For instance, can one verify that the tunneling particles originated predominantly near the peak of the wave packet? Can one, alternatively, determine the length of time a particle spends (on average) under the barrier? This "sojourn" or "dwell" time is a quantity which had been of much interest to the condensed-matter community, as it would allow one to describe the importance of interactions between a tunneling particle and the surrounding environment, and the validity of approximations such as adiabatic following. Even those who were not troubled by the superluminal peak delay presumed that the physical time spent in a given region of space would have to be greater than or equal to $d / c$; a number of models of the interaction between a tunneling particle and the environment were used to support this conjecture and yield "interaction times" for the tunneling problem(Büttiker and Landauer, 1985).

In (Steinberg, 1995a b) , I applied the ideas of weak measurement to this question, and was surprised. On the one hand, no weak measurement would show the supposed "bias" towards the leading edge of the incident wave packet. Furthermore, one could rewrite the tunneling "interaction time" as a time-integral of the probability to be in the barrier, which in turn decomposed into a probability density at each position and time:

$$
\begin{aligned}
\tau & \equiv \int_{-\infty}^{\infty} d t P_{\mathrm{bar}}(t) \\
& =\int_{-\infty}^{\infty} d t \int_{0}^{d} d x|\Psi(x, t)|^{2} .
\end{aligned}
$$

By generalizing this to the case of postselected subensembles (i.e., calculating the weak values of the projector $\delta(\hat{X}-x)$ for various positions $x$ ), it proved possible to derive a "conditional probability distribution" for a particle to be at position $x$, given that it was prepared in a state 
$|i\rangle$ (incident on the barrier from the left, in a given wave packet) and detected in a final state $|f\rangle$ (transmitted to the far side of the barrier). The time $\tau$ turned out to be in general complex, but its real part - that part which describes the position shift of a pointer coupled to the particle's presence in the barrier region - is of the same order of magnitude as the group delay, and exhibits the same "superluminal" features. A plot of the evolving conditional-probability distribution is shown in Figure 3.

One of the striking things about this figure is that the particle appears to spend essentially no "time" (in the sense of the real part of a weak value) near the centre of the barrier. A reflected particle only spends time within an exponential decay length of the input facet; while a transmitted particle spends roughly equal amounts of time near the entrance and near the exit (as one might have surmised from the symmetry of the experimental arrangement, or of the formula for weak values). Figure 4 presents a gedankenexperiment to elucidate the physical meaning of these curves. Consider a proton constrained to tunnel in one direction. It tunnels along a series of holes in parallel conducting sheets, which serve to break the tunnel barrier up into a sequence of electricallyshielded regions. As described in the context of the three-box problem above, one way to measure the weak value of a "probability" (or of its time-integral, a dwell time) is to study the momentum shift of a test charge which interacts with the particle in question. Here we imagine an electron, initially at rest, between each pair of conducting plates. We measure the final momentum of each electron after the passage (reflection or transmission) of the proton, sorting according to whether the proton was transmitted or reflected. On each event, by definition of a weak measurement, the electrons' momenta are far too uncertain to draw any conclusions (or else the presence of the electrons would so perturb the motion of the proton that there would be no sense in discussing it as a tunneling problem; see (Steinberg, 1999a)). After averaging over the momenta found for numerous transmitted protons, however, one would find the symmetric distribution indicated in the figures.

In keeping with our intuitions, but not with the standard (timeasymmetric) recipe for dealing with quantum evolution and measurement, we see that in addition to concluding from the initial condition (a particle approaching the barrier from the left) that the wave packet penetrates roughly one exponential decay length into the left side of the barrier, one may conclude from the final condition (a particle exiting the barrier on the right, for instance) that it had penetrated one decay length into the right side of the barrier as well. Weak measure- 
ments allow us to discuss the behaviour of "to-be-transmitted" particles and "to-be-reflected" particles separately, and observe that even when described by the same initial wave function, they may have different physical effects on weakly coupled environments.

It turns out that one of the popular approaches to tunneling times, the Larmor time (Büttiker, 1983), is in essence nothing but a weak value. This time has two different components, whose individual physical meanings were obscure, however, until reinterpreted in the light of this new formalism. It is now clear that they correspond to the real and imaginary parts of the weak measurement, and that the former corresponds to the pointer shift (the measurement result as extrapolated from the classical limit), while the latter indicates a necessary back-action of the particle due to the measurement, which can be made arbitrarily small by using a sufficiently weak measurement.

One question raised by the evolving conditional probability distributions plotted above is whether, in the superluminal-tunneling regime, the particle really does move from a wave packet on the left of the barrier to one on the right in a time shorter than $d / c$, without spending significant time in the centre of the barrier. While we all know that a cause cannot have any measurable effect at a spacelike separated point, is it perhaps possible for a single particle to have an effect at two points spacelike separated from one another (but not from the source of the particle) (Steinberg, 1998c)? Clearly, it suffices for two people on opposite sides of a radio transmitted to listen to the same broadcast, for a cause to have two spacelike-separated effects. But is a single quantum particle truly as nonlocal as this radio wave? We all know that if a strong measurement is made of the position of a photon, it can no longer be found in a different position. But since repeated weak measurements can be made on the same wave function, and are not modified by the action of other weak measurements made at the same time, I was led to suspect that it should be possible to weakly measure the probability of a tunneling particle passing through a region of spacetime which contains the bulk of the incident wave packet, as well as the probability of the same particle passing through the (spacelike separated) region which contains the bulk of the transmitted wave packet. If conditioned on eventual transmission of the tunneling particle, both of these would be close to unity - on average, each individual particle would have had an effect on two spacelike-separated detectors. Figure 5 shows a spacetime diagram for the experiment under consideration (Steinberg, 1998b; Steinberg et al., 1998a). An energy-filter is added after transmission, to 
"erase" (Scullv et al., 1991; Kwiat et al., 1992) any information about the time of arrival of the transmitted peak; without this filter, the possibility of a strong measurement of the time of arrival of the particle would preclude any possibility that it had come from the initial peak.

We have been setting up an experiment (Steinberg et al., 1998a, 1999b) to observe laser-cooled atoms tunneling through an optical barrier, wherein probes interacting with atoms at various positions and various points in time should allow us to study the weak-measurement predictions. In parallel, we have been thinking about the theoretical approach necessary to determine whether each single particle had actually affected two measurement apparatuses at spacelike separation, or whether despite this appearance on average, each particle could be thought of as being at only one device at a time. If the wave function is not merely a measure of our ignorance, but in some deeper sense "real," then one ought perhaps not to be surprised by a particle having a (weak) effect in two places at the same time, so long as no "collapse" occurs. Nevertheless, I believe that most physicists still have an underlying intuition about the indivisibility of particles which would lead them to predict such effects could not occur. Amusingly, when I have tried to explain our proposed experiments, most of the physicists $I$ know, who are willing to discuss such things, had the opposite reaction: of course a particle can be in two places at the same time, and of course both pointers may shift simultaneously!

Our initial proposal was to build on the following idea. Consider pointers $P 1$ and $P 2$ at spacelike separated positions. We would like to demonstrate that even though each picks up only a small shift on a single event, it is possible to show that individual particles interacted with both pointers. Let us therefore assume the opposite, the corpuscular hypothesis that on a given event, either $P 1$ or $P 2$ was affected, but not both. Nevertheless, weak measurements will show that both $P 1$ and $P 2$ are shifted on average by an amount roughly equal to unity (a measurement that the particle was almost certainly in a given region). This must imply that on some occasions, $P 2$ is unshifted, while on other occasions, it is shifted by an amount greater than unity; and the same for $P 1$. Due to the anticorrelation of these shifts, we expect the distribution of the difference $P 1-P 2$ to develop a larger uncertainty. If the uncertainty of $P 1-P 2$ did not grow, we would conclude that the shifts of $P 1$ and $P 2$ were not anticorrelated, and that each individual particle must really have interacted with both.

While some work has been started on higher-moment weak values Iannaccone, 
1996), this field is far from mature. We decided that a simple approach would be to use the same measuring device at $P 1$ and $P 2$, but with equal and opposite signs. For instance, using the Larmor-clock approach, a magnetic field along $+z$ at region $P 1$ could couple to the electron's spin so long as the particle was in that region, while a magnetic field along $-z$ at region $P 2$ could couple to the same spin with the opposite sign. The rotation of the spin in the $x-y$ plane would automatically record the difference between $P 1$ and $P 2$. It is straightforward to show, in the limit of very weak measurements and narrow-band energy filters, that the effects of the two magnetic fields should cancel perfectly. All the transmitted particles should have their spin unaffected, implying that they were affected equally by the two interaction regions. This would, I thought, support the hypothesis that quantum particles can truly be in two places (and have measurable effects there) at the same time.

More recently, consideration of the three-box problem described above led me to carry out the same calculation in that situation. Spin rotations of opposite sign in arms $A$ and $B$ would also cancel out, implying that the particle was really in both $A$ and $B$ simultaneously. Yet we saw earlier that the joint probability for being in $A$ and $B$ was in fact zero. One can go through the same argument in the tunneling case. Even though the conditional probability distribution does fill both regions $P 1$ and $P 2$, the product of projection operators onto two spacelike separated regions automatically vanishes (in the Heisenberg picture), because these regions constitute orthogonal subspaces of Hilbert space. It now seems that even in the case of superluminal tunneling, a true weak measurement of the joint probability of being in two places at once is always guaranteed to yield zero. Thus even though $\langle P 1\rangle_{\mathrm{wk}}=\langle P 2\rangle_{\mathrm{wk}}$ and $\langle P 1-P 2\rangle_{\mathrm{wk}}=0$, one can show

$$
\begin{aligned}
\left\langle(P 1-P 2)^{2}\right\rangle_{\mathrm{wk}} & = \\
& =\left\langle P 1^{2}\right\rangle_{\mathrm{wk}}+\left\langle P 2^{2}\right\rangle_{\mathrm{wk}}-\langle P 1 P 2\rangle_{\mathrm{wk}}-\langle P 2 P 1\rangle_{\mathrm{wk}} \\
& ==1+1+0+0=2 .
\end{aligned}
$$

If one treats this as the definition of the uncertainty in a weak value, one certainly finds anticorrelations: $P 1$ and $P 2$ only shift by unity at the expense of their difference growing uncertain by $\sqrt{2}$, just as though they had shifted in an entirely uncorrelated fashion. On the other hand, if one simply calculates the final state of a transmitted spin which was subject to equal and opposite interactions at $P 1$ and $P 2$, one finds no increase in the uncertainty of its orientation. Further work will be nec- 
essary to determine what weak values can really teach us about nonlocality, and how best to define the uncertainties and correlations of these probabilities which are not bounded by the usual classical rules. Nevertheless, it is apparent that weak measurements allow us to discuss postselected systems (such as tunneling particles) in a much more powerful way than was possible in the more conventional language of evolving and collapsing wave functions. In the meantime, we continue to build our laser-cooling experiment, to verify these predictions, and to study generalisations which occur when "real" measurements (i.e., decoherence or dissipation) are introduced, and when the "weakness" of an interaction is varied.

\subsection{Quantum information and postselection}

In the burgeoning field of quantum information(Nielson, 2000), it is well known that photons are excellent carriers of quantum information, easily produced, manipulated, and detected, and relatively immune to "decoherence" and undesired interactions with the surrounding environment. This has led to their widespread application in quantum communications (Bennett and Brassard, 1984; Brendel et al., 1999; Buttler et al., 2000). Unfortunately, the superposition principle of linear optics implies that different photons behave independently of one another- without some nonlinearity, it is impossible for one photon to influence the evolution of another photon, and this has long made it seem that optics would be an unsuitable platform for designing a quantum computer. Even certain straightforward projective measurements, such as the determination of which of the four Bell states $\dagger$ a photon pair is in, prove to be intractable without significantly stronger nonlinearities than exist in practice(Mattle et al., 1996; Bouwmeester et al., 1997; Calsamiglia and Lütkenhaus, 2001). Much work has focussed on developing exotic systems such as cavity-QED experiments (Nogues, 1999: Turchette et al., 1995) in which enhanced nonlinearities allow for the design of effective quantum logic gates, while most of quantumcomputation research has instead focussed on using atoms, ions, or solids to store and manipulate "qubits" Cirac and Zoller, 1995; Monroe et al., 1995: Kane, 1998). Recently, it was noted that detection itself is a

$\dagger$ the maximally-entangled polarisation states of two particles: $|H V\rangle \pm|V H\rangle$ and $|H H\rangle \pm|V V\rangle$ in the case of photons, or equivalently, $|J=0, m=0\rangle$, $|J=1, m=0\rangle$, and $|J=1, m=1\rangle \pm|J=1, m=-1\rangle$ for a pair of spin- $1 / 2$ particles. 
nonlinear process, and that appropriately chosen postselection may be used to "mimic" the kinds of optical nonlinearity one would desire for the construction of an optical quantum logic gate Knill et al., 2001; Pittman et al., 2001). In parallel, work has continued on searches for systems in which true optical nonlinearities might be enhanced by factors on the order of $10^{9}$ or $10^{10}$, as would be necessary for the construction of fundamental logic gates Franson, 1997; Harris and Hau, 1999; Kash et al., 1999).

We recently showed that it is possible to use quantum interference between photon pairs to effectively enhance nonlinearities by a similar order of magnitude. Using a crystal of BBO, beta-barium borate, it is possible to frequency-double a beam of light, converting two photons at $\omega$ into one photon at $2 \omega$ with some small $\left(\mathcal{O}\left(10^{-10}\right)\right)$ probability, or alternatively to "down-convert" a photon at $2 \omega$ into a pair of photons around $\omega$, with equally low probability (Steinberg et al., 1996). These effects are extremely common and extremely important in modern nonlinear optics, but rely on high-intensity beams to generate significant effects; two individual photons entering such a crystal would have a negligible interaction. For this reason, one experiment which purported to perform " $100 \%$ efficient" quantum teleportation by using a nonlinear interaction to carry out the necessary Bell-state determination actually needed to replace one of the incident photons with a beam containing billions of identical copies (Kim et al., 2001). By contrast, we discovered that adding an additional pump beam (with billions of photons) to the system leads to a quantum-interference effect which can enhance the interaction between two single-photon-level beams by many orders of magnitude. In (Resch et al., 2001b), we show that this can lead to $>50 \%$-efficient frequency-doubling of photon pairs. This effect is closely related to earlier work on quantum suppression of parametric downconversion by Anton Zeilinger's group (Herzog et al., 1994).

The basic scheme is shown in Figure 6. Two beams at $\omega$, each containing less than 1 photon on average, enter a nonlinear crystal; these beams are conventionally known as "signal" and "idler." Simultaneously, a strong pump beam at $2 \omega$ pumps the crystal in a mode which couples to signal and idler via the interaction Hamiltonian

$$
\mathcal{H}=g a_{p}^{\dagger} a_{s} a_{i}+\text { h.c. . }
$$

This can convert a single pump photon into a signal-idler pair, or vice versa, albeit with vanishingly small efficiency. The three input beams are in coherent states, and thus the initial state of the system may be written 
$|\Psi\rangle=\left|\alpha_{p}\right\rangle_{p}\left|\alpha_{s}\right\rangle_{s}\left|\alpha_{i}\right\rangle_{i}$. For weak inputs $\left|\alpha_{s}\right|,\left|\alpha_{i}\right| \ll 1$, but a strong classical pump $\left(\left|\alpha_{p}\right|^{2} \sim 10^{10}\right)$, the interaction can be controlled such that to lowest order, all photon pairs are removed from the signal and idler beams (i.e., they are up-converted into the pump mode, although this effect is too weak to be directly observed). This occurs due to destructive interference between the amplitude for a photon pair to be present in $s$ and $i$, and the amplitude for a pump photon to down-convert into the same modes (Resch et al., 2002a). Importantly, this interference effect depends on the relative phase of the three beams, which means that it cannot work if any of the beams has a well-defined photon number, since the optical phase and the photon number are incompatible observables (roughly speaking - on the same order of roughness as the time-energy uncertainty principle $-\Delta n \Delta \phi \geq 1 / 2$ ).

This upconversion effect can be thought of as a highly efficient switchif a photon happens to be present in the signal mode, than no photon in the idler mode can be transmitted; and vice versa. Unfortunately, this is only true if it is fundamentally unknown whether the signal mode possessed a photon or not. By observing the absence of coincidence counts after the device, we may conclude that any photon pairs which had been present disappeared... but on no individual occasion did we know a photon pair actually existed!

We extended this work to a geometry more closely related to one of the standard logic gates of quantum information theory, the controlledphase gate (Nielson, 2000; Resch et al., 2002b). Still relying on interference between incoming photon pairs and the down-conversion process, we altered the relative phase so that the probability of a photon pair emerging was not significantly altered, but its quantum phase would be shifted relative to that of the vacuum or a single photon in either beam alone. To measure this, we built the homodyne setup in Figure 7. This can be thought of as a simple Mach-Zehnder interferometer for a signal photon (really a signal beam with an average photon number per pulse much less than 1). Into one arm of the interferometer, our pumped crystal is inserted. At the same time, a "control" beam is sent through the crystal's idler mode. If a control photon is present, then a phase shift is impressed on any passing signal photon; this is observed as a shift in the Mach-Zehnder interference pattern (see Fig. 8). We were able to observe shifts as large as $\pm 180^{\circ}$, or very small phase shifts with little effect on the probability itself, depending on the strength of the pump beam relative to that of the signal and control beams. Once more, however, to operate this gate, we had to operate in a condition 
of ignorance. We send in beams which may or may not have photons, but when we observe a "control" photon leaving the crystal, we find the desired effect on the signal. Can we conclude that this postselection determined that there had been a control photon there all along, and that the logic gate performed the correct operation for an input of logical ' 1 '? To understand the operation of the gate - the phase shift imprinted on the signal beam - it is necessary to take into account both the state preparation (the well-defined phase differences between the beams) and the postselection (the presence of a control photon).

In a manner somewhat reminiscent of the KLM scheme (Knill et al., 2001), this requires a fundamental change in the way one thinks about logic operations, with inputs being determined not by preparing the appropriate state, but by postselecting the desired value of the input (Resch et al., 2002c). So far, it remains unclear how widely such effects could be applied in quantum information; we do not presently know of a way to incorporate them into the standard paradigm of quantum computing. On the other hand, we have shown(Resch et al., 2001a) that despite its eccentricity and potential pitfalls, this "conditional-phase switch" can indeed be used to implement the Bell measurements which were previously impossible for individual photon pairs, provided only that the photon pairs are produced in the appropriate superposition with vacuum. For subtle but important reasons, this means our technique cannot be used for unconditional quantum teleporation; but it can be used to improve earlier experiments on subjects such as quantum dense coding (Mattle et al., 1996).

While it is not possible to have a well-defined phase and a well-defined photon number in a quantum state, it is possible to prepare one and postselect the other: and weak measurements show us that at intermediate times, the system possesses some characteristics of both the initial and final states. It seems that weak measurement may be precisely the formalism needed for describing such enhanced nonlinearities, and probably a broader range of "nondeterministic" operations currently being investigated in quantum logic.

\subsection{Having your cake and eating it too}

There is another example of a possible application for these enhanced nonlinearities, and we are presently setting up an experiment to demonstrate this. In 1992, Lucien Hardy proposed an ingenious quantum paradox which involved intersecting electron and positron interferom- 
eters, wherein colliding electrons and positrons would undergo certain annihilation (Hardv, 1992a b). Of course, this scheme was quickly recognized to be something of a stretch experimentally, and it was hoped that the experiment could be performed with optical interferometers instead. Unfortunately, as mentioned several times already, the interaction between different photons is so weak in practical systems that the equivalent of an "annihilation" event - an upconversion event, for instance was exceedingly rare. A mathematically equivalent paradox was eventually tested optically (White et al., 1999), but no direct demonstration of the original conundrum has been possible to date.

Hardy's paradox relies on the concept of "interaction-free measurements" introduced by Elitzur and Vaidman (Elitzur and Vaidman, 1993). Briefly, it is possible to set up an interferometer as in Figure 9 to transmit all the input light out one port, known as the "bright" port. Ideally, no photon should ever be detected at the "dark" port. However, any object which blocks one of the paths of the interferometer will destroy the interference, and therefore generate some probability of a photon exiting the dark port. Clearly, in the cases in which a photon is observed at this port, one can conclude that (A) it was not blocked by the object; but (B) the object must have been in place (since without the object, interference prevents any counts from being observed there). In the original example, this made it possible to achieve the surprising feat of confirming that an infinitely sensitive bomb was functioning - without setting it off. In later work (Kwiat et al., 1995), it was shown that this task could be accomplished with arbitrarily high efficiency, through ingenious modifications to the interferometer.

Although such measurements are popularly referred to as "interactionfree," in some quantum mechanical sense, they clearly do involve an interaction: a "bomb" initially in an uncertain position may be collapsed into the interferometer arm, through the detection of a photon at the dark port. Such considerations motivated the extension of the problem to two overlapping interaction-free-measurement (IFM) interferometers, shown in Figure 10, each of which can be thought of as measuring whether or not the other interferometer's particle is in the "in" path. The reasoning now is simple. If an electron interferometer and a positron interferometer overlap at "in," in such a way that the electron and positron are certain to annihilate if they meet there, then each particle may serve to "block" the other particle if and only if it takes the "in" path. If each interferometer is aligned so that all electrons reach $B_{e}$ and all positrons $B_{p}$, then these two interferometers are 
IFMs. An electron will only be detected at $D_{e}$ if the positron was in the way. Similarly, a positron can only reach $D_{p}$ if an electron is in the way. Naturally, if both the electron and the positron are at "in," then they annihilate, and cannot be observed. For this reason, one should never observe an electron at $D_{e}$ and a positron at $D_{p}$ at the same time.

Yet this is not the case. Quantum mechanically, there is a finite probability for both the electron and the positron to reach their dark ports. How do we interpret this? The conventional answer is that we have learned the error of our classical ways. While the IFM was able to tell us whether or not a classical particle was blocking one arm of an interferometer, we transgressed by drawing counterfactual conclusions about a quantum particle which was not directly observed. Clearly, this is not a very satisfying state of affairs, but perhaps it is true that quantum mechanics does not allow us to make "retrodictions" of the sort we rely on to construct this paradox.

Despite the clear contradiction with classical reasoning, the astute reader may recall that at least in the case of weak measurements, classical intuition often works surprisingly well, albeit at the expense of certain other intuitions, such as the positive-definiteness of probabilities. Indeed, it was recently pointed out (Aharonov et. al, 2002) that weak measurements can "resolve" the paradox raised by Hardy. How is this? Consider weak measurements of the probabilities for the various particles to be in the various arms of the interferometer, and of the corresponding joint probabilities. From where does the apparent paradox arise? If we post-select on cases where both photons reach the dark port, we want to conclude that the probability of the electron having followed the "in" path, $P\left(e^{-}\right.$in $)=1$; and also that $P\left(e^{+}\right.$in $)=1$. So far so good, except that we also believe that $P\left(e^{-}\right.$in and $e^{+}$in $)$must $=0$, since both particles would have annihilated had they met along the "in" path. Of course, we have already seen a similar situation in Table 1. Just because $\mathrm{A}$ and $\mathrm{B}$ both happen with certainty (in a weak-measurement sense) does not imply that $\mathrm{A}$ and $\mathrm{B}$ ever happen simultaneously. Aharonov et al. calculate that the above probabilities do in fact hold, and that to satisfy the various sum rules, the probability of one particle being "in" and the other being "out" is $100 \%$, and that the probability of both particles being "out" is $-100 \%$. In this sense, there is no more paradox. All the paths can be measured simultaneously and in arbitrary combinations, so long as the measurements are all weak. And given this proviso, all our expectations from intuitive analysis of the IFMs should prove to be correct. The price we need to pay for this resolution is to accept that, 
at least in situations of postselection, certain probabilities may turn out to be negative.

Although we still do not know how to turn our "switch" into a quantum computer, recall that it allows us to cause photon pairs to upconvert with nearly unit efficiency. This is the analog of the $e^{+} e^{-}$annihilation in Hardy's original formulation, and we can now hope to observe his paradox directly, using a coherently-driven nonlinear crystal as the interaction region for "annihilating" our photon pairs. Now this switch, which had the disturbing property of working only in a "nondeterministic," after-the-fact manner, becomes the ideal tool for studying the difficult situations one gets into when trying to make retrodictions about quantum-mechanical systems.

\subsection{Conclusion}

In this rapid tour of a variety of recent (and future) experiments and theoretical investigations, I have tried to focus some attention on the new trend towards attempting to talk about history in quantum mechanics, and in particular to talk about the history of specific subensembles defined by both state preparation and postselection. The formalism of weak measurements addresses such problems in a very natural fashion, but yields all manner of counterintuitive predictions. At the same time, it has an unshakable connection to real measurements which could be (and often are) performed in the laboratory; I describe certain experiments now in progress which should further demonstrate the fruitfulness of this formalism. The relationship between weak measurements and generalized probability theories appears to be particularly strong, but more work remains to be done to elucidate the meaning of these exotic (negative, or even complex) quantities which obey many of the axioms of probability theory. In particular, weak measurements provide one with a little more leeway than orthodox quantum mechanics when it comes to describing what the state of a system "really was" between preparation and detection, but in so doing, raises a variety of difficult questions, especially relating to the reality of the wave function, and the nonlocality of individual quantum particles. It is interesting to note that a variety of experiments, ranging from new concepts for quantum computation to cavity-QED studies of open-system quantum dynamics, have recently provoked increased interest in the mathematical description of post-selected subensembles. Perhaps the time is finally right for mainstream quantum physicists to attack these problems, and in the 
process develop a better understanding of the nature of space, time, and measurement in quantum mechanics.

\subsection{Acknowledgments}

I would like to acknowledge my coworkers at Toronto - Kevin Resch, Jeff Lundeen, Stefan Myrskog, Jalani Fox, Ana Jofre, Chris Ellenor, Masoud Mohseni, and Mirco Siercke - both for their efforts in the lab and for their ideas, many of which have found their way into this chapter. I would also like to thank Ray Chiao and Paul Kwiat for their collaboration and useful discussions over many years. Finally, for thought-provoking conversations about weak measurements I would like to thank Howard Wiseman, Jeff Tollaksen, Sandu Popescu, Lev Vaidman, Avshalom Elitzur, Gonzalo Muga, Markus Büttiker, and Jeeva Anandan, in addition of course to Yakir Aharonov, whose seminars first introduced me to the concept. Some of the work described in this chapter was supported by NSERC, by Photonics Research Ontario, by the Canadian Foundation for Innovation, and by the US Air Force Office of Scientific Research under the QuIST programme (F49620-01-1-0468). 


\section{Bibliography}

Aharonov, Y., Albert, D.Z. and Vaidman, L. (1988). Phys. Rev. Lett. 60, 1351.

Aharonov, Y. and Vaidman, L. (1990). Phys. Rev. A 41, 11.

Aharonov, Y. and Vaidman, L. (1991). J. Phys. A 24, 2315.

Aharonov, Y., Popescu, S., Rohrlich, D. and Vaidman, L. (1993). Phys. Rev. A 48, 4084.

Aharonov, Y. and Anandan, J. (1993). Phys. Rev. A 47, 4616..

Aharonov, Y., Botero, A., Popescu, S., Reznik, B. and Tollaksen, J. (2001). Phys. Lett. A 301, 130.

Aharonov, Y. and Vaidman, L. (2002). In Time in Quantum Mechanics, ed. J.G. Muga et al., Springer-Verlag.

Aharonov, Y. and Vaidman, L. (2002). How One Shutter Can Close N Slits. Preprint quantum-ph/0206074

Büttiker, M. and Landauer, R. (1982). Phys. Rev. Lett. 49, 1739.

Büttiker, M. (1983). Phys. Rev. B27, 6178.

Büttiker, M. and Landauer, R. (1985). Phys. Scr. 32.

Bennett, C.H. and Brassard, G. (1984). Proc. IEEE Int. Conf. on Comp. Sys. and Sig. Proc., Bangalore, India, 175.

Bell, J.S. (1987). Speakable and Unspeakable in Quantuum Mechanics, Princeton University Press.

Bouwmeester, D., Pan, J.-W., Mattle, K., Eible, M., Weinfurter, H. and Zeilinger, A. (1997). Nature 390, 575.

Brendel, J., Gisin, N., Tittel, W. and Zbinden, H. (1999). Phys. Rev. Lett. 82, 2594 .

Buttler, W.T. et al. (2000). Phys. Rev. Lett. 84, 5652.

Chu, S. and Wong, S. (1982). Phys. Rev. Lett. 48, 738.

Cirac, J.I. and Zoller, P. (1995). Phys. Rev. Lett. 74, 4091.

Chiao, R.Y. and Steinberg, A.M. (1997). Tunneling Times and Superluminality. In Progress in Optics Vol. 37, ed. E. Wolf, Elsevier.

Calsamiglia, J. and Lütkenhaus, N. (2001). Appl. Phys. B 72, 67.

Enders, A. and Nimtz, G. (1993). J. Phys. I France 3, 1089.

Elitzur, A.C. and Vaidman, L. (1993). Found. Phys. 23, 987.

Feynman, R.P. (1987). In Quantum Implications, eds. B.J. Hiley and F. David Peat, Routledge and Kegan Paul.

Franson, J.D. (1997). Phys. Rev. Lett. 78, 3852. 
Foster, G.T., Orozco, L.A., Castro-Beltran, H.M. and Carmichael, H.J. (2000). Phys. Rev. Lett. 85, 3149.

Garrett, C.G.B. and McCumber, D.E. (1970). Phys. Rev. A 1, 305.

Hauge, E.H. and Støvneng, J.A. (1989). Rev. Mod. Phys. 61, 917.

Hardy, L. (1992). Phys. Lett. A 167, 17.

Hardy, L. (1992). Phys. Rev. Lett. 68, 2981-2984.

Herzog, T.J., Rarity, J.G., Weinfurter, H. and Zeilinger, A. (1994). Phys. Rev. Lett. 72, 629.

Harris, S.E. and Hau, L.V. (1999). Phys. Rev. Lett. 82, 4611.

Iannaccone, G. (1996) Weak measurement and the traversal time problem. Adriatico Research Conference on Tunneling and Its Implications, ICTP, Trieste. Preprint quant-ph/9611018

Kwiat, P.G. , Steinberg, A.M. and Chiao, R.Y. (1992). Phys. Rev. A 45, 7729.

Kwiat, P.G., Weinfurter, H., Herzog, T., Zeilinger, A. and Kasevich, M.A. (1995). Phys. Rev. Lett. 74, 4763.

Kane, B.E. (1998). Nature 393, 133.

Kash, M.M. et al. (1999). Phys. Rev. Lett. 82, .

Kim, Y.-H., Kulik, S.P. and Shih, Y. (2001). Phys. Rev. Lett. 86, 1370.

Knill, E., Laflamme, R. and Milburn, G. (2001). Nature 409, 46.

Liebfried, D., Meekhof, D.M., King, B.E., Monroe, C., Itano, W.M. and . Wineland, D.J. (1996). Phys. Rev. Lett. 77, 4281.

MacColl, L.A. (1932). Phys. Rev. 40, 621.

Muckenheim, W. and et al. (1983). Phys. Rep. 133, 339.

Monroe, C., Meekhof, D.M., King, B.E., Itano, W.M. and Wineland, D.J. (1995). Phys. Rev. Lett. 75, 4714.

Mattle, K., Weinfurter, H., Kwiat, P.G. and Zeilinger, A. (1996). Phys. Rev. Lett. 76, 4656.

von Neumann, J. (1955). Mathematical Foundations of Quantum Mechanics, Princeton Univ. Press.

von Neumann, J. (1983). In Quantum Theory and Measurement, eds. J. A. Wheeler and W. H. Zurek, Princeton University Press.

Nielsen, M.A. and Chuang, I.L. (2000). Quantum Computation and Information, Cambridge University Press.

Nogues, G. et al. (1999). Nature 400, 239.

Pitowski, I. (1982). Phys. Rev. Lett. 48, 1299.

Pittman, T.B. , Jacobs, B.C. and Franson, J.D. (2001). Phys. Rev. A 64, 062311.

Resch, K.J., Lundeen, J.S. and Steinberg, A.M. (2001). To appear in the Proceedings of the 2001 Solvay conference; preprint quant-ph/0204034

Resch, K.J., Lundeen, J.S. and Steinberg, A.M. (2001). Phys. Rev. Lett. 87, 123603.

Resch, K.J., Lundeen, J.S. and Steinberg, A.M. (2002). J. Mod. Opt. 49, 487.

Resch, K.J., Lundeen, J.S. and Steinberg, A.M. (2002). Phys. Rev. Lett. 89, 037904 .

Resch, K.J., Lundeen, J.S. and Steinberg, A.M. (2002). Phys. Rev. Lett. 88, 113601.

Reznik, B. and Aharonov, Y. (1995). Phys. Rev. A 52, 2538.

Ritchie, N.W.M., Story, J.G. and Hulet, R.G. (1991). Phys. Rev. Lett. 66, 1107.

Scully, M.O., Englert, B.-G. and Walther, H. (1991). Nature 351, 111. 
Scully, M.O., Walther, H. and Schleich, W. (1994). Phys. Rev. A 49, 1562.

Spielmann, C., Szipöcs, R., Stingl, A. and Krausz, F. (1994). Phys. Rev. Lett. 73, 2308.

Steinberg, A.M., Kwiat, P.G. and Chiao, R.Y. (1993). Phys. Rev. Lett. 71, 708.

Steinberg, A.M. and Chiao, R.Y. (1994). Phys. Rev. A 49, 2071.

Steinberg, A.M. (1995). Phys. Rev. Lett. 74, 2405.

Steinberg, A.M. (1995). Phys. Rev. A 52, 32.

Steinberg, A.M., Kwiat, P.G. and Chiao, R.Y. (1996). In the American Institute of Physics Atomic, Molecular, and Optical Physics Handbook, ed. G.W.F. Drake, AIP Press.

Steinberg, A.M., Myrskog, S., Moon, H.S., Kim, H.A., Fox, J. and Kim, J.B. (1998). Ann. Phys. (Leipzig) 7, 593.

Steinberg, A.M. (1998). Superlattices and Microstructures 23, 823.

Steinberg, A.M. (1998). Found. Phys. 28, 385.

Steinberg, A.M. (1999). J. Kor. Phys. Soc. 35, 122.

Steinberg, A.M., Myrskog, S., Moon, H.S., Kim, H.A., Fox, J. and Kim, J.B. (1999). In Mysteries, Puzzles, and Paradoxes in Quantum Mechanics, ed. R. Bonifacio, AIP Conference Proceedings 461, 36.

Steinberg, A.M. (2000). Phys. World 13, 21.

Turchette, Q.A., Hood, C.J., Lange, W., Mabuchi, H. and Kimble, H.J. (1995). Phys. Rev. Lett. 75, 4710.

Unruh, W.G. (1994). Phys. Rev. A 50, 882.

Wigner,E.P. (1932). Phys. Rev. 40, 749.

Wigner, E.P. (1995). Phys. Rev. 98, 145.

White, A.G., James, D.F.V., Eberhard, P.H. and Kwiat, P.G. (1999). Phys. Rev. Lett. 83, 3103.

Wang, L.J., Kuzmich, A. and Dogariu, A. (2000). Nature 406, 277.

Wiseman, H.M. (2002). Phys. Rev. A. 65, 032111.

\subsection{Figure Captions}

(i) A two-slit experiment. When a particle is fired from a source towards the double slit, we can use Schrödinger's equation to predict its state as it passes the two slits: a symmetric wave function localized equally behind both slits. But when a particle appears at one point on the screen, what can we conclude about its history? As we all know, we cannot state it went through one slit or the other. Shall we say it went through both with equal likelihoods, as determined by the state preparation? Or from the location of the spot on the screen, can we construct some more accurate wave function? Can we just use Schrödinger's equation to propagate the electron backwards in time? This would discard all information about the state preparation, which seems extreme. Yet to discard all information about the future may also be unnecessary- for instance, even the claim of a symmetric 
double-peaked wave function only made sense given the knowledge that the particle did make it through the double-slit to eventually reach the screen, knowledge only obtained via postselection.

(ii) a. The quantum 3-box problem. If a particle is hidden in three boxes in a superposition $(A+B+C) / \sqrt{3}$, but is subsequently found to be in the (different but nonorthogonal) superposition $(A+B-C) / \sqrt{3}$, what can one say about the state of the particle while in the box?

b. Experimental schematic for an optical implementation of the three-box problem. Photons are sent into a 3-rail interferometer, with the three rails playing the roles of boxes $\mathrm{A}, \mathrm{B}$, and $\mathrm{C}$. A $\pi$ phase shift is introduced in rail $\mathrm{C}$, such that detection at the camera post-selects a superposition $(A+B-C) / \sqrt{3}$. To weakly "measure" the particle in one or another of the boxes, small transverse displacements are induced in each of the rails, and an image of the postselected photon distribution is taken to determine the size of the effects of displacements in each of the boxes.

(iii) The time-evolution of the "weak" conditional probability distribution for a particle's position as it tunnels through a barrier. The heavy curve shows the real part of this distribution (the magnitude of the expected measurement result), while the dashed curve shows its imaginary value (the "back-action" due to measurement), and the light curve shows the distribution for reflected particles (essentially equal to $|\Psi|^{2}$ ). Note that at early and late times, the weak distribution mimics the full incident or transmitted wave packet, while at intermediate times it has an exponentially small magnitude inside the forbidden region.

(iv) A gedankenexperiment using distant electrons to measure how much time a tunneling proton spends in each of several shielded regions of space. While the proton is between a given pair of conducting plates, only the corresponding electron feels a significant force. After the tunneling event, the momentum shift of each electron thus records the amount of time spent by the proton between the plates in question. The implication of weak-measurement theory is that reflected protons only transfer momentum to electrons near the entrance (a), while transmitted protons affect electrons 
near both edges of the barrier (b). Electrons in the center only undergo a position shift, related to the back-action of the measurement.

(v) A gedanexperiment to investigate whether or not a subset of tunneling particles may truly prove to have "been" in two places at the same time, due to the superluminal group velocity in tunneling. The peak of the transmitted gaussian may emerge at a point spacelike separated fromm the peak of the incident gaussian. An energy filter is necessary to "erase" any timing information which would preclude the detected particle from having been present at the incident peak; once a particle is transmitted through a narrowband filter, information about its time of origin is smeared out.

(vi) The two-photon "switch" experiment: quantum interference between photon pairs being generated through down-conversion and already being present in two laser beams can lead to nearly unitefficiency upconversion of photon pairs from classical beams.

(vii) The two-photon switch incorporated into a Mach-Zehnder interferometer serves to demonstrate a conditional-phase gate, i.e., cross-phase modulation at the single-photon level.

(viii) Fringe patterns observed at the output of the Mach-Zehnder when a trigger photon was detected (black circles; solid line), versus when no trigger photon was detected (white squares; dashed line). A significant phase shift is observed on the signal beam due to the presence of a single photon in the trigger mode.

(ix) A Mach-Zehnder interferometer as proposed by Elitzur and Vaidman for performing "interaction-free measurements." When the path lengths are balanced, all photons reach the "bright" port and none the "dark" port. An absorbing object placed inside the interferometer may cause photons to reach the dark port, indicating the presence of the object even though those photons could (in some sense) never have interacted with the object directly.

(x) Two overlapping interaction-free measurement devices ("IFMs," in the jargon) implement Hardy's Paradox. One device is an 
electron interferometer, and the other a positron interferometer. They overlap at $\mathrm{W}$, where it is supposed that an electron and a positron arriving simultaneously will annihilate with certainty. If one can truly conclude from electron detection at $D_{-}$that the positron was in the interaction region $\mathrm{W}$, and from positron detection at $D_{+}$that the electron was in $\mathrm{W}$, then one should never see coincident detections between the two dark detectors, since the particles would have annihilated at W. Quantum mechanics shows that this is not the case. 

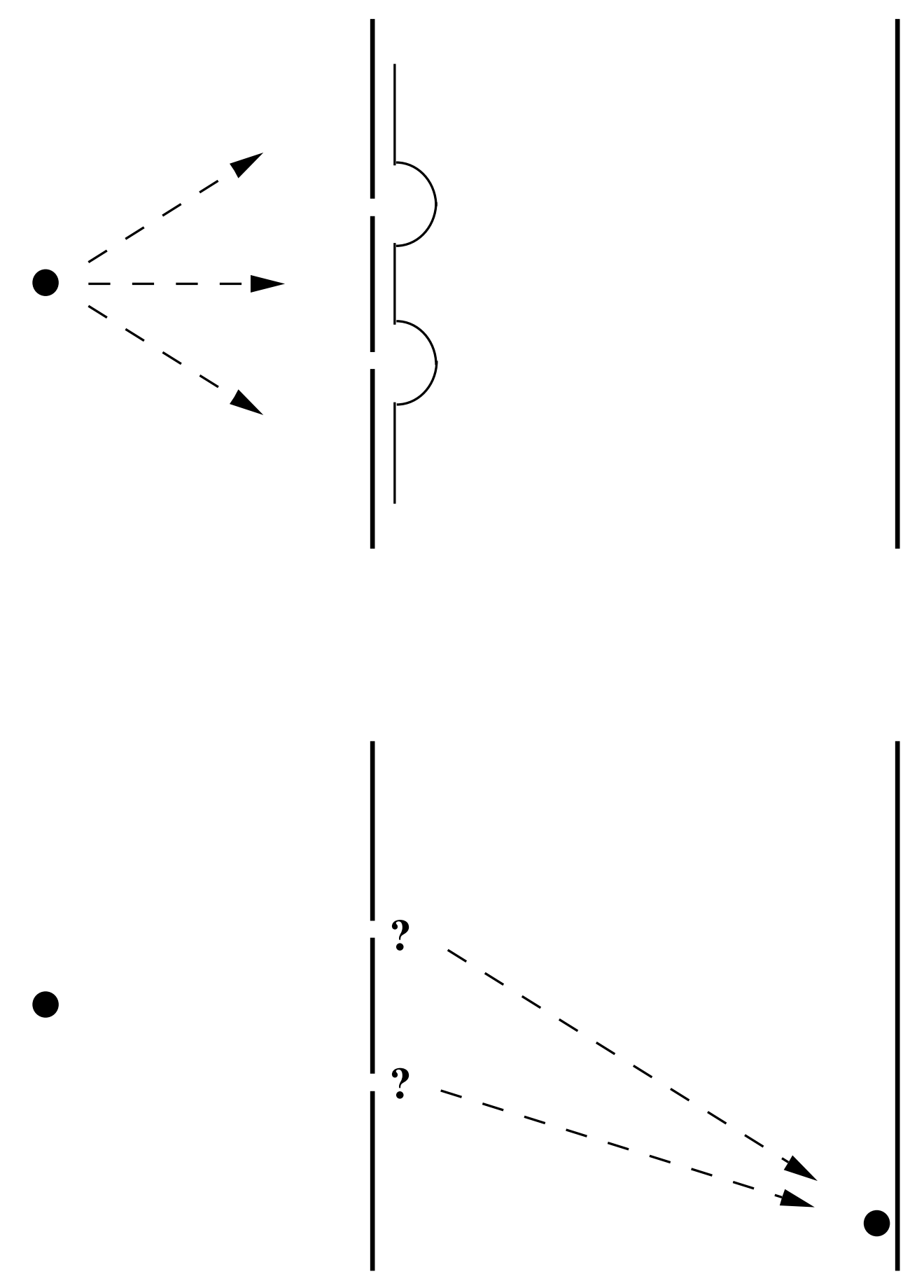


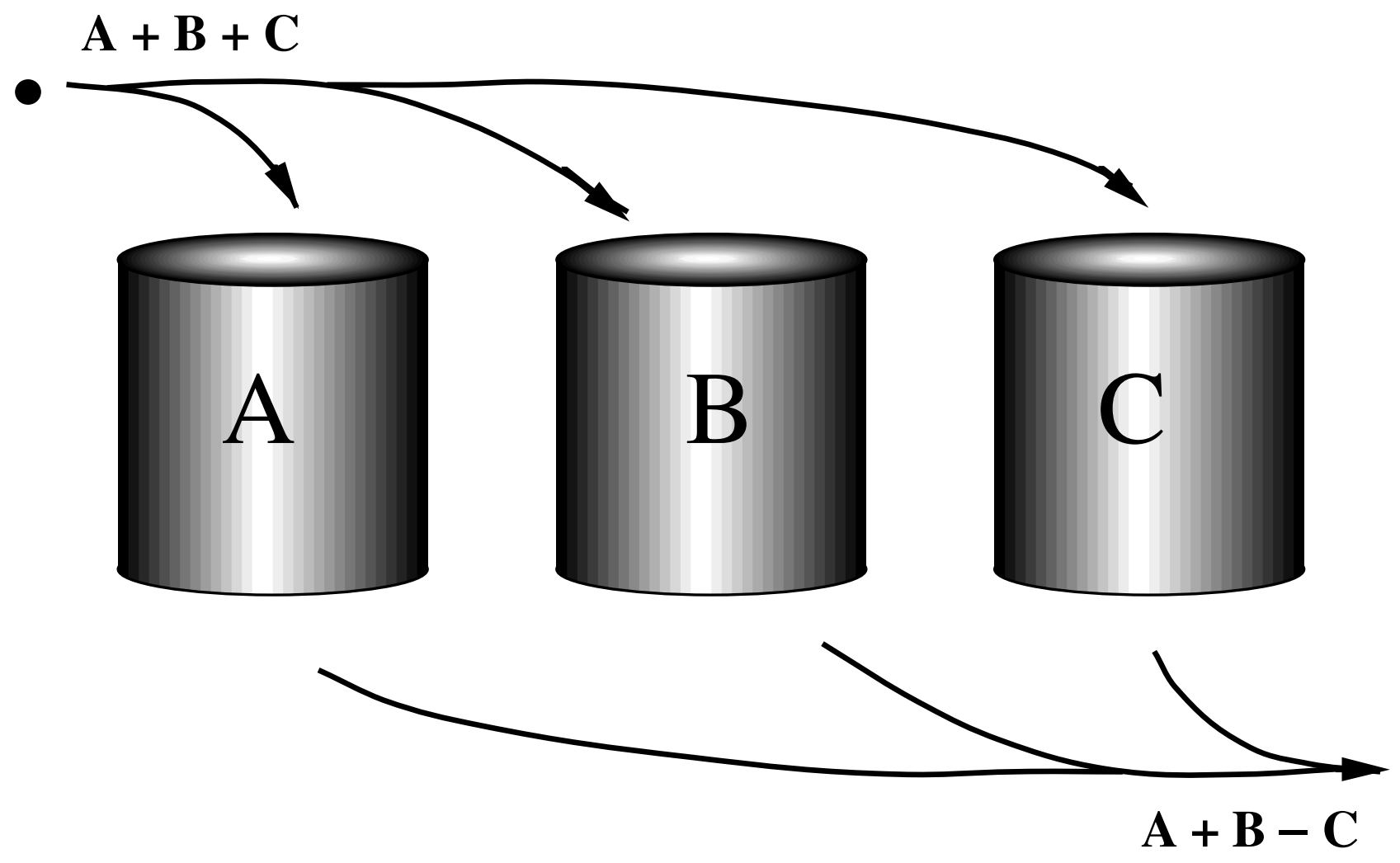




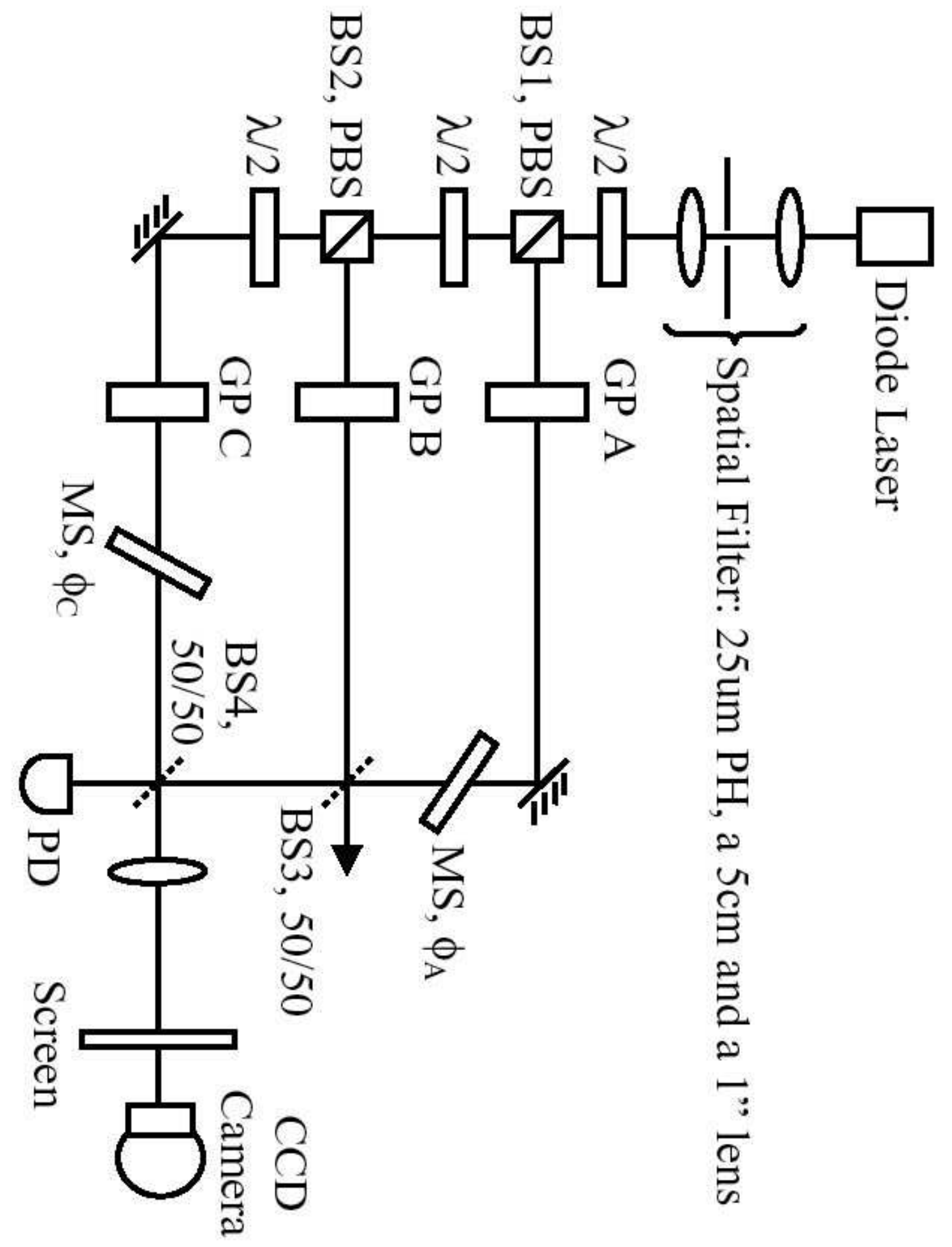




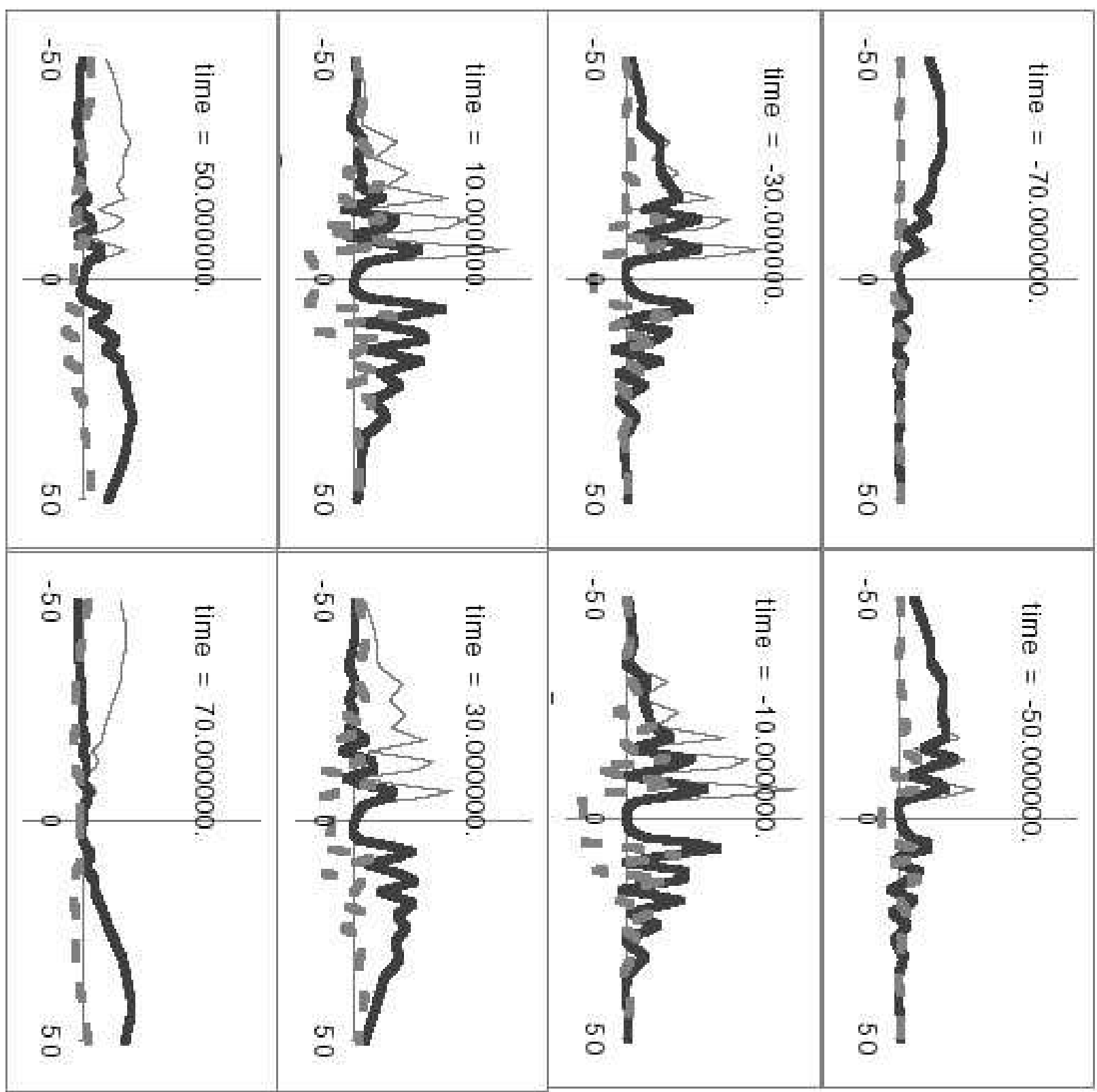




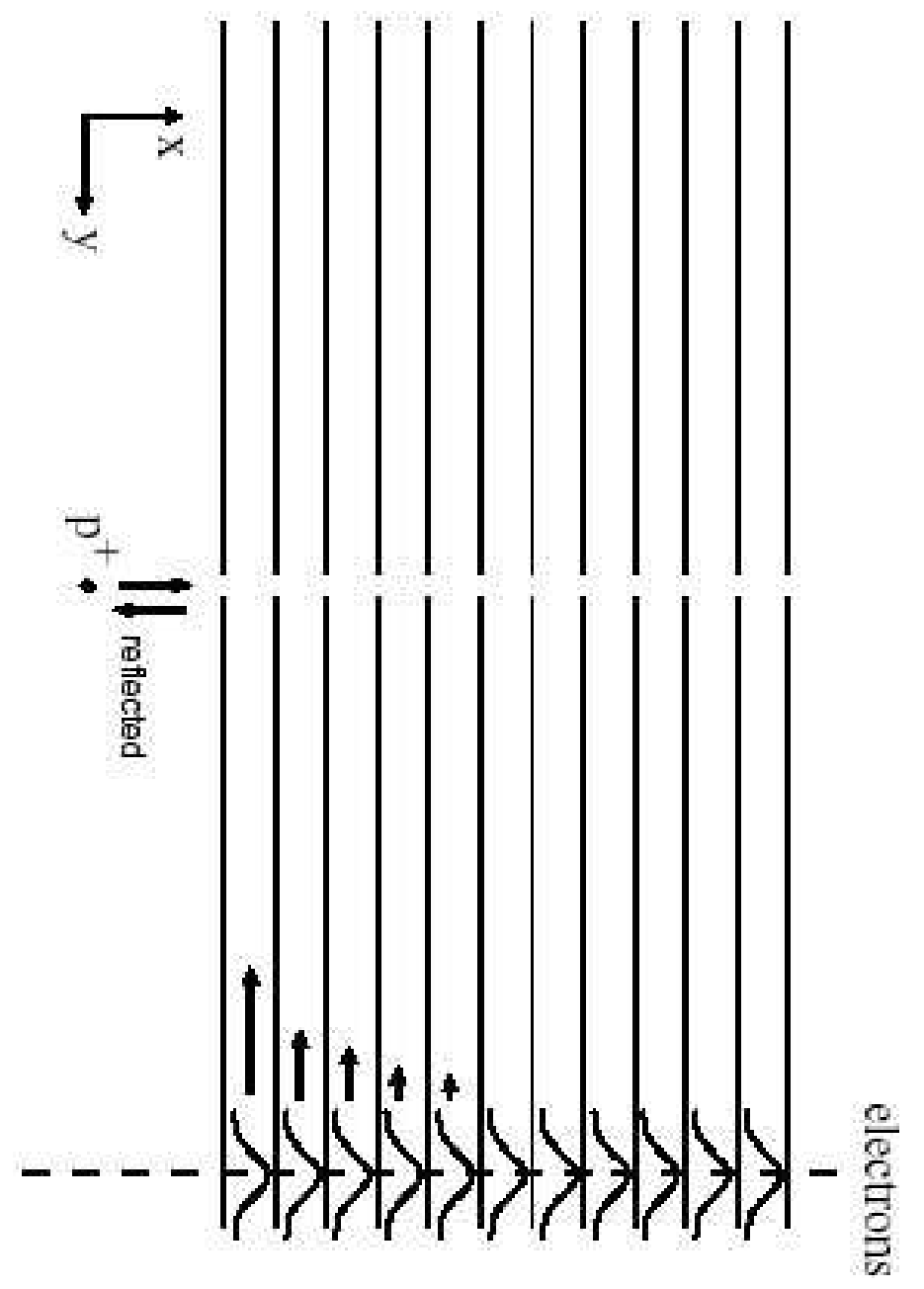




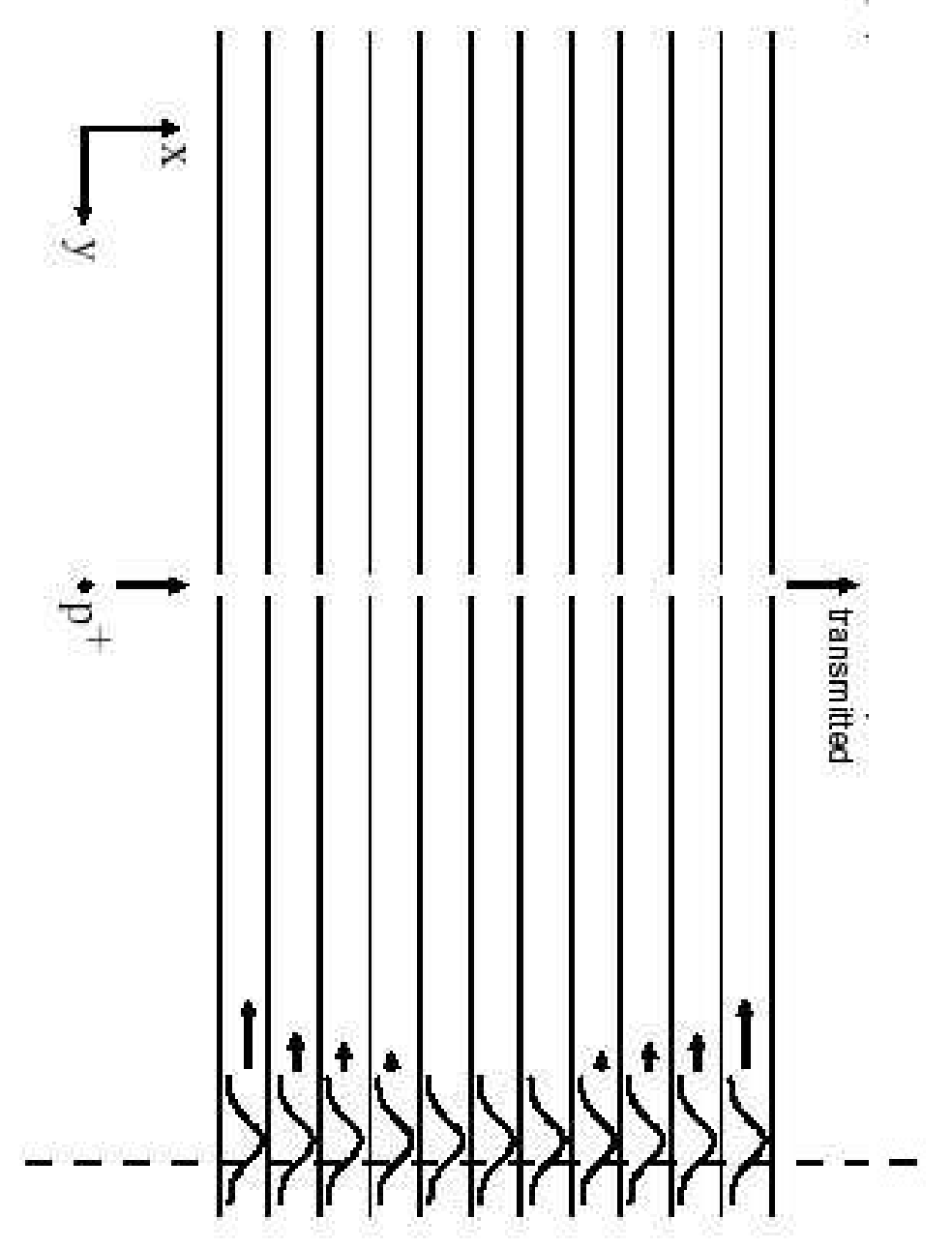




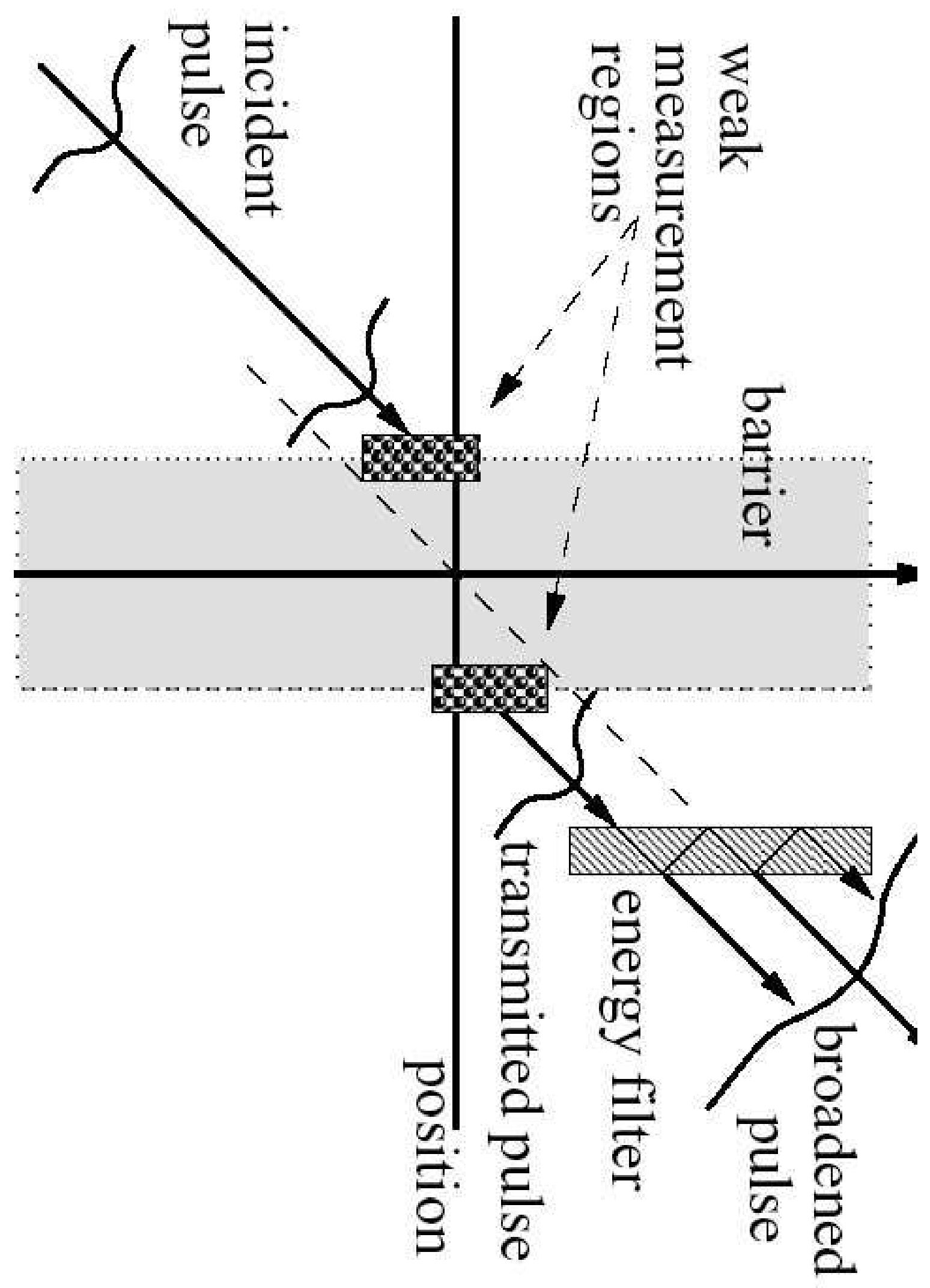




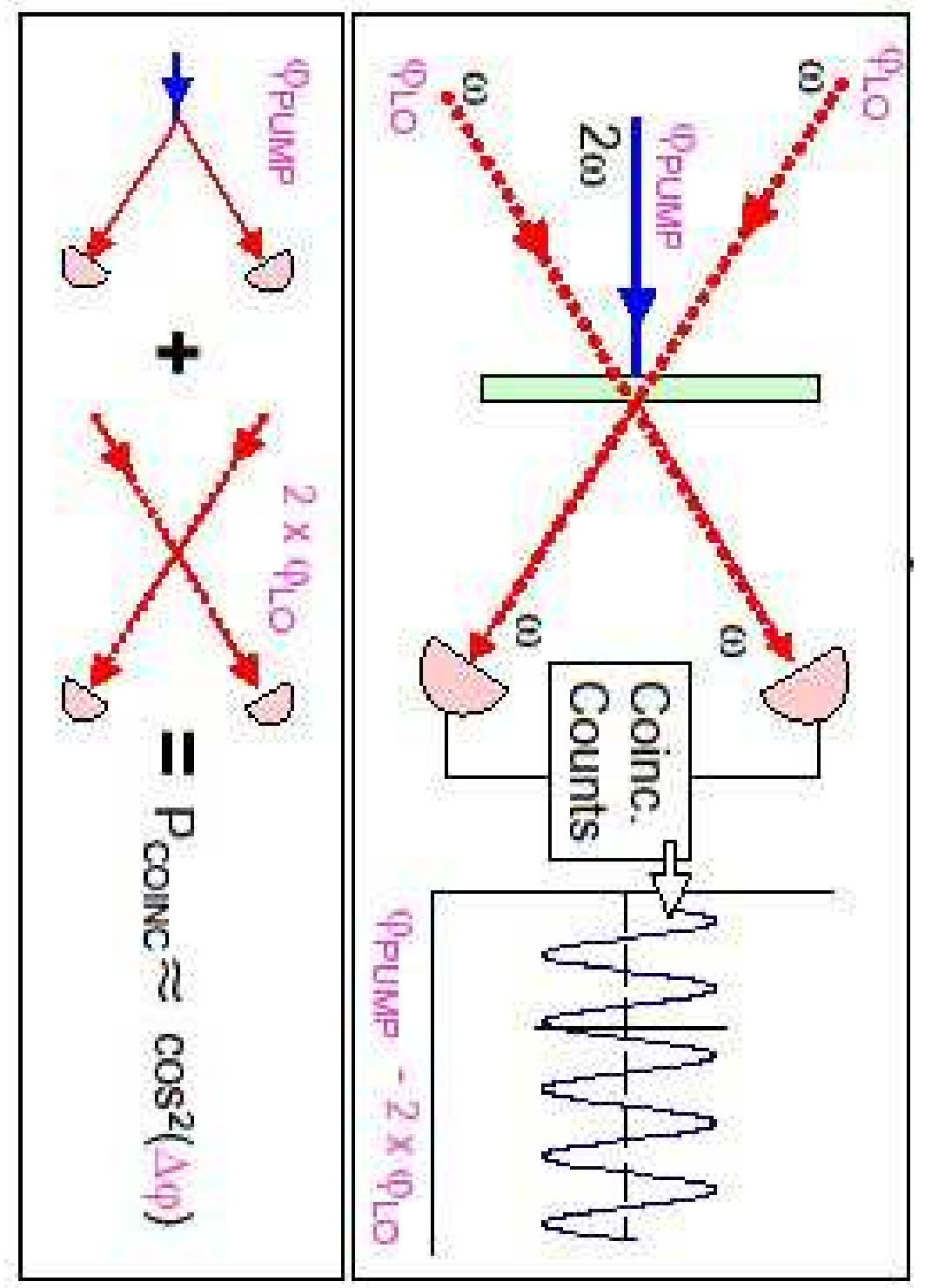




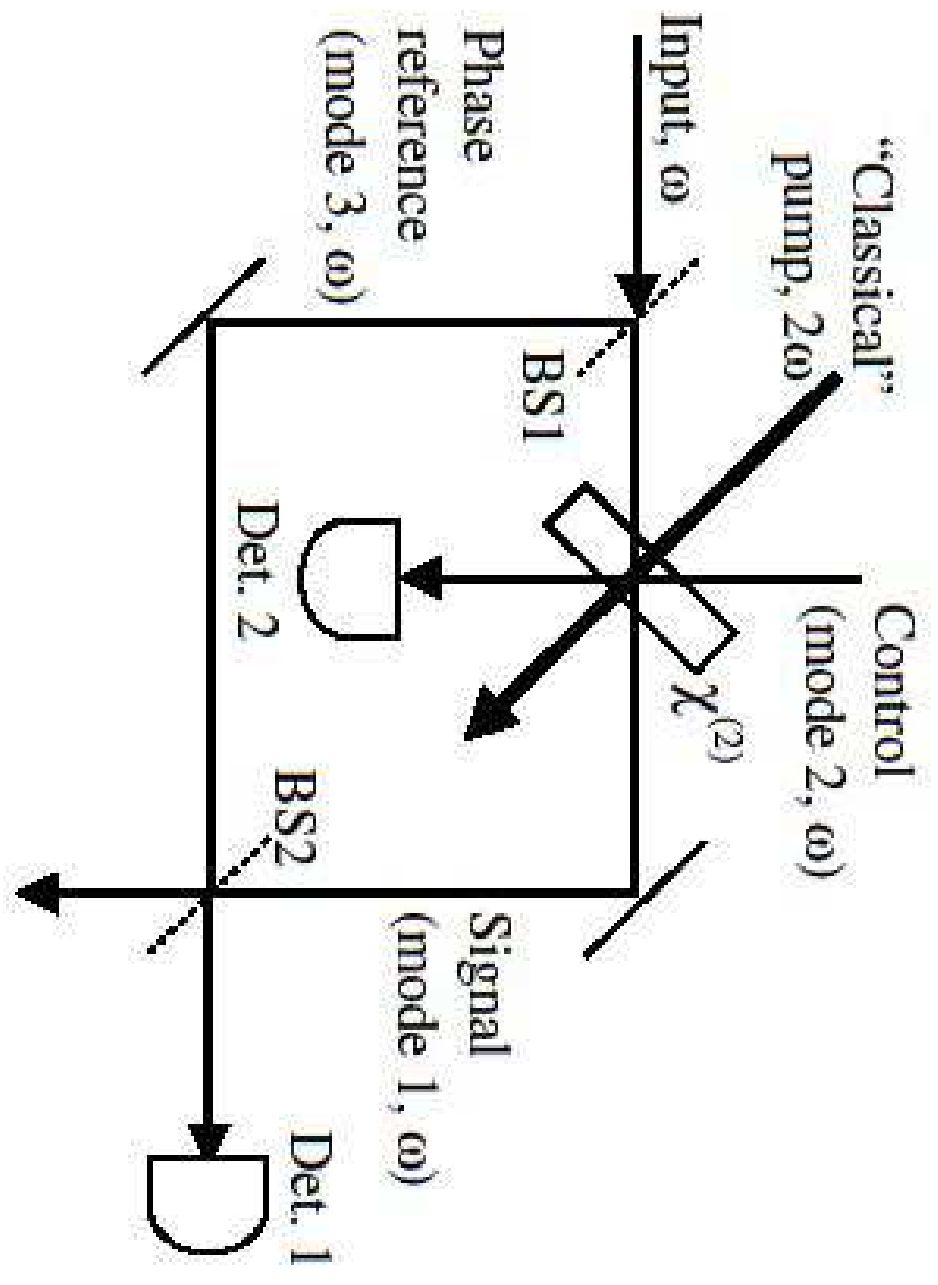


Coincidence Counts (/20s)

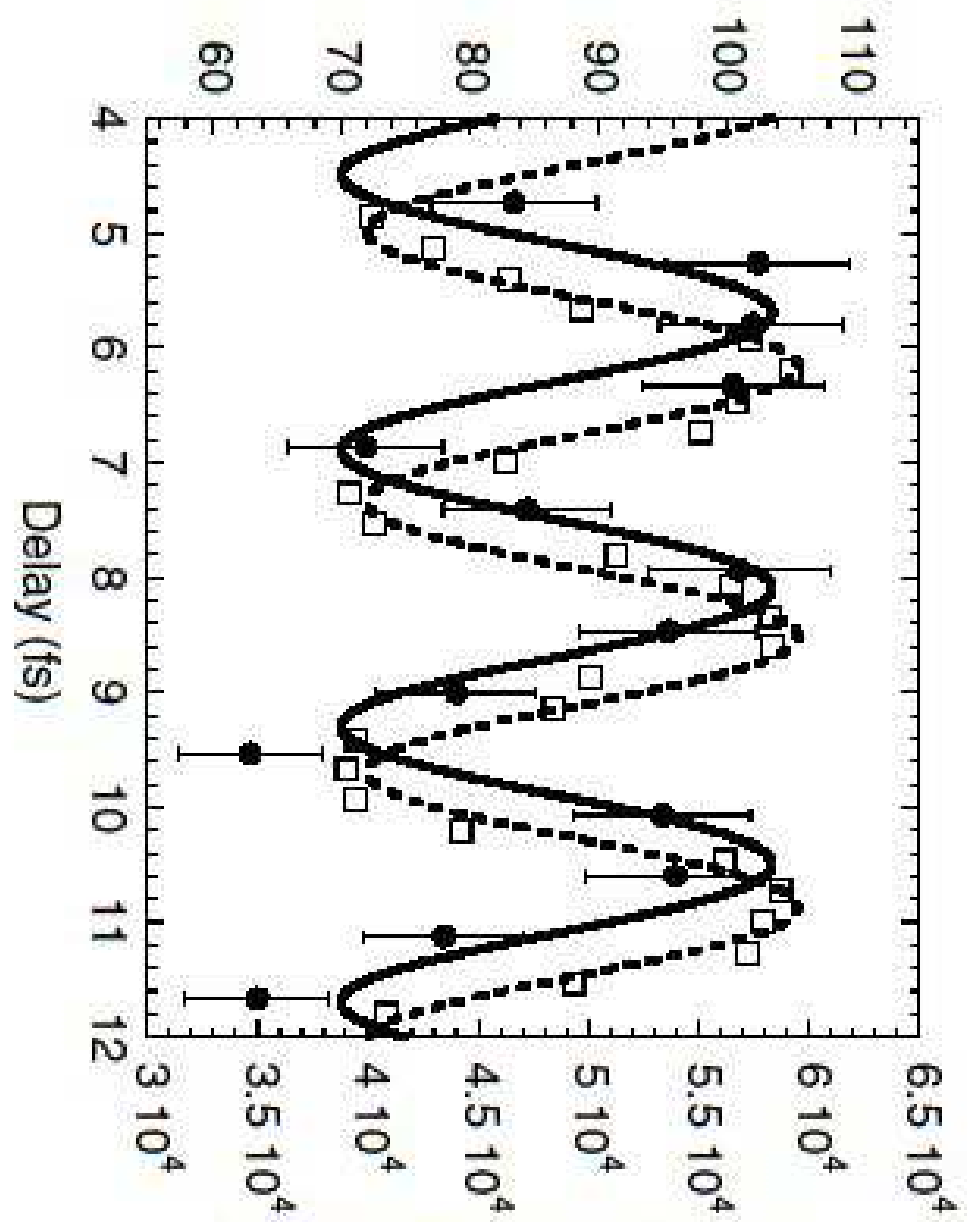

(s0z/) sә|6u!s әu/pomoH 


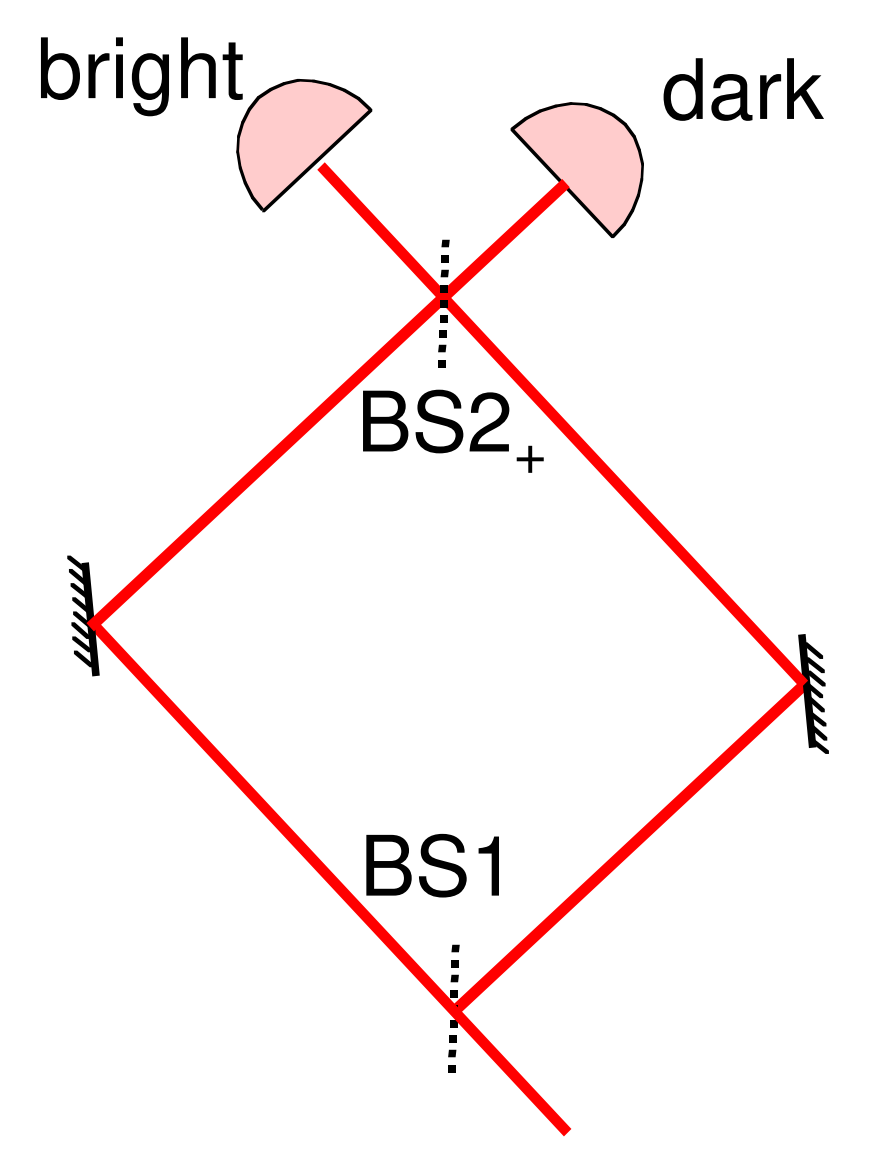




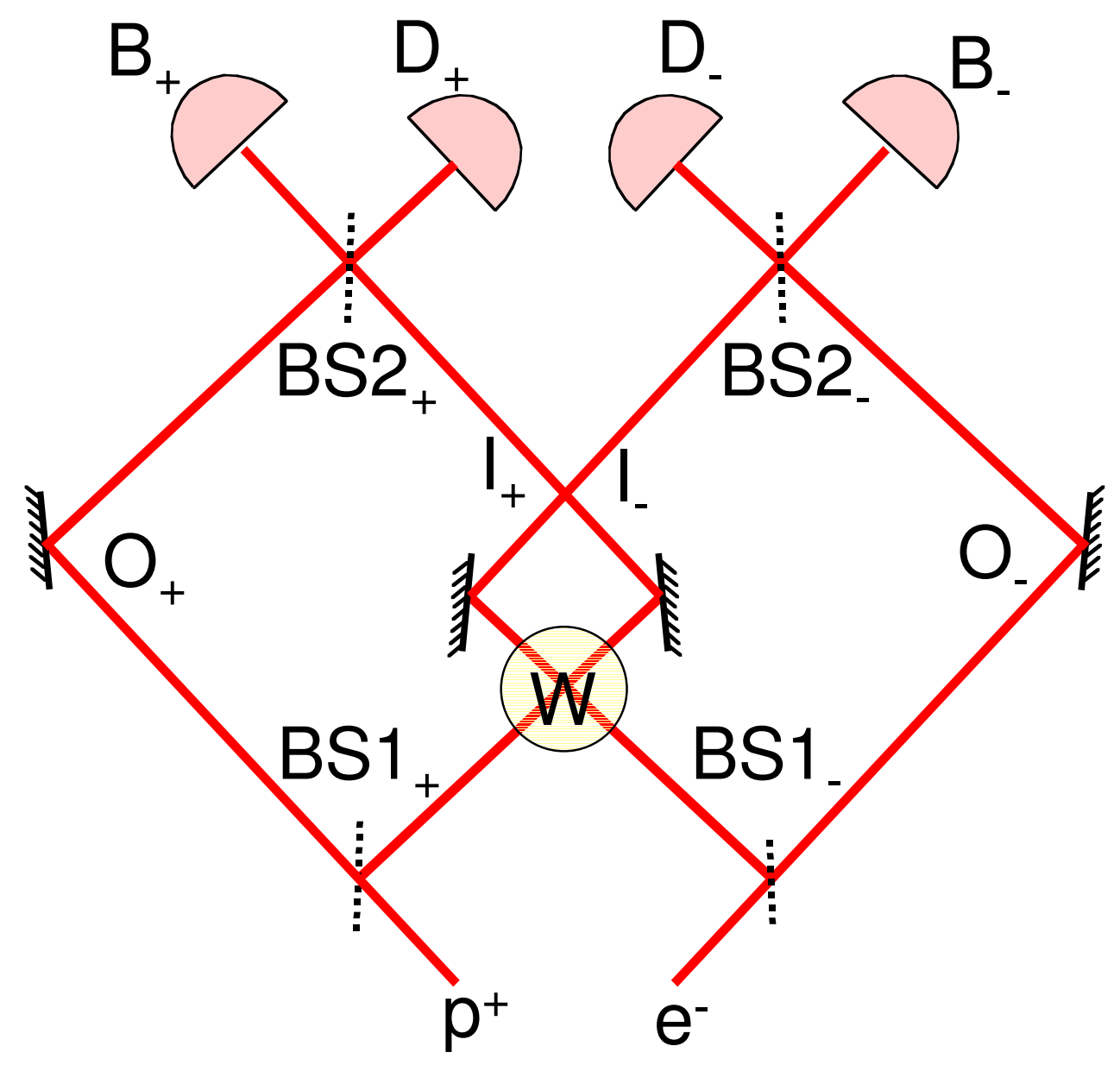

